TRANSACTIONS OF THE

AMERICAN MATHEMATICAL SOCIETY

Volume 354, Number 5, Pages 1921-1945

S 0002-9947(02)02924-0

Article electronically published on January 8, 2002

\title{
SMALL RATIONAL MODEL OF SUBSPACE COMPLEMENT
}

\author{
SERGEY YUZVINSKY
}

\begin{abstract}
This paper concerns the rational cohomology ring of the complement $M$ of a complex subspace arrangement. We start with the De ConciniProcesi differential graded algebra that is a rational model for $M$. Inside it we find a much smaller subalgebra $D$ quasi-isomorphic to the whole algebra. $D$ is described by defining a natural multiplication on a chain complex whose homology is the local homology of the intersection lattice $L$ whence connecting the De Concini-Procesi model with the Goresky-MacPherson formula for the additive structure of $H^{*}(M)$. The algebra $D$ has a natural integral version that is a good candidate for an integral model of $M$. If the rational local homology of $L$ can be computed explicitly we obtain an explicit presentation of the ring $H^{*}(M, \mathbf{Q})$. For example, this is done for the cases where $L$ is a geometric lattice and where $M$ is a $k$-equal manifold.
\end{abstract}

\section{INTRODUCTION}

This paper concerns the cohomology ring of the complement of a complex subspace arrangement. Let $\mathcal{A}$ be a finite set of proper subspaces of a finite dimensional complex linear space $V$. Put $C(\mathcal{A})=V \backslash \bigcup_{A \in \mathcal{A}} A$. We can assume that there are no inclusions among elements of $\mathcal{A}$. A rough characterization of $\mathcal{A}$ is given by the lattice $X=X(\mathcal{A})$ consisting of all the intersections of the elements from $\mathcal{A}$ ordered opposite to inclusion and labeled by their complex codimensions.

If each subspace $A \in \mathcal{A}$ is a hyperplane, then $\mathcal{A}$ can be given by a set $\left\{\alpha_{A}\right\}_{A \in \mathcal{A}} \subset$ $V^{*}$ where $\operatorname{ker} \alpha_{A}=A$. In this case, the lattice $X$ is geometric and the codimension of each of its elements can be recovered from the ordering as the rank of this element. There are two main results describing the structure of the $\operatorname{ring} R=H^{*}(C(\mathcal{A}), \mathbb{Z})$ in this case. The first one is the Arnold-Brieskorn theorem [1, 4] stating that the algebra of differential forms generated by the closed forms $\frac{1}{2 \pi i} \frac{d \alpha_{A}}{\alpha_{A}}, A \in \mathcal{A}$, is isomorphic to $R$ under the de Rham homomorphism. The second one is the Orlik-Solomon theorem stating that $R$ depends only on $X$, that is, on the set of intersections of hyperplanes and its partial order by (opposite) inclusion. Moreover, the theorem gives a presentation of $R$ by generators and relations defined by $X$.

In the general case, $X$ is not necessarily geometric and the codimensions cd $A$ of $A \in X$ cannot be recovered anymore from the order on $X$. Again, there are two main results about $H^{*}(C(\mathcal{A}))$. The first one is the Goresky-MacPherson theorem 9] stating that the groups $H^{p}(C(\mathcal{A}))$ are determined by the labeled lattice $X$. More precisely these groups are expressed in terms of the local homology groups of $X(\mathcal{A})$

Received by the editors November 13, 2000

2000 Mathematics Subject Classification. Primary 52C35, 05 E25.

(C)2002 American Mathematical Society 
as follows. For every $p$

$$
\widetilde{H}^{p}(C(\mathcal{A}), \mathbb{Z})=\bigoplus_{A \in X} \widetilde{H}_{2 \operatorname{cd} A-p-2}((V, A), \mathbb{Z})
$$

where $(V, A)=\{B \in X \mid V<B<A\}$ and $H_{*}((V, A))$ is the homology of the complex of flags in $(V, A)$. This is an example of application of Stratified Morse theory developed in [9]. Later, this result was obtained by a different method in [16]. A completely different approach was used recently by De Concini and Procesi [5]. They constructed a rational model for $C(\mathcal{A})$ using only the labeled lattice $X$ (see also section 2) and proved that the rational cohomology algebra and rational homotopy type of $C(\mathcal{A})$ are defined by this lattice. The natural problem that was left open is how to recover the ring structure explicitly from the combinatorics. In particular how to relate the multiplication to the local homology of $X$ that occurs in the Goresky-MacPherson formulas.

The present paper solves this problem (Theorem 6.6). To be more specific we need to introduce more notation here. For every pair $(A, B)(A, B \in X)$ denote by $A \vee B$ the join (i.e., the least upper bound) of the pair. The joins of sets of atoms from $(V, A) \cup(V, B)$ form a subposet $X_{A, B}$ of $(V, A \vee B)$. The following condition on $A, B$ is an important breaking point

$$
\operatorname{cd}(A \vee B)=\operatorname{cd} A+\operatorname{cd} B .
$$

If this condition holds, then the flag complex of $X_{A, B}$ has the homotopy type of the suspension of the join of the flag complexes of $(V, A)$ and $(V, B)$. Then there is a natural isomorphism

$$
\varphi_{A, B}: \widetilde{H}_{r}((V, A), \mathbb{Q}) \otimes \widetilde{H}_{s}((V, B), \mathbb{Q}) \rightarrow \widetilde{H}_{r+s+2}\left(X_{A, B}, \mathbb{Q}\right) .
$$

Theorem. The multiplication in $H^{*}(C(\mathcal{A}), \mathbb{Q})$ is given by bilinear pairings

$$
\psi_{A, B}: \widetilde{H}_{r}((V, A), \mathbb{Q}) \otimes \widetilde{H}_{s}((V, B), \mathbb{Q}) \rightarrow \widetilde{H}_{r+s+2}((V, A \vee B), \mathbb{Q})
$$

for every $A, B \in X$. Here $\psi_{A, B}$ is the composition of $\phi_{A, B}$ with the embedding $X_{A, B} \subset(V, A \vee B)$ if (因) holds and $\psi_{A, B}=0$ otherwise.

Notice that the set of flag complexes of $X(\mathcal{A})$ for all the subspace arrangements $\mathcal{A}$ includes the homotopy types of all finite simplicial complexes. In this generality, it is impossible to give any explicit description of local homology classes of $X(\mathcal{A})$ and the multiplication on them. Instead we describe a multiplication on chain groups of the local flag complexes that induces the right multiplication on the homology.

Let $A, B \in X(\mathcal{A})$ with condition (荬. Let $F_{A}=\left(A_{1}<A_{2}<\cdots<A_{i}\right)$ and $F_{B}=\left(B_{1}<B_{2}<\cdots<B_{j}\right)$ be flags in the open intervals $(V, A)$ and $(V, B)$ respectively. Augment the flags by $A$ and $B$ respectively and take the shuffle product of them. The result is a linear combination (with coefficients equal to signs of permutations) of certain sequences of $A_{r}$ and $B_{s}$. Create a flag from each such sequence taking the intersections of all initial subsequences. Equate flags with repetitions to 0 and delete $A \vee B$ at the ends of all others. The resulting linear combination of flags is the product $F_{A} \cdot F_{B}$. This product generates the product on linear combinations of flags by bilinearity that commutes with the differentials. Hence it induces a bilinear pairing

$$
\widetilde{H}_{i-1}((V, A)) \otimes \widetilde{H}_{j-1}((V, B)) \rightarrow \widetilde{H}_{i+j}((V, A \vee B))
$$


This is the multiplication on local homology of $X$. The multiplication can be defined even easier on so-called Whitney (or relative atomic) complexes but we will present it elsewhere.

For the classes of lattices whose local homology can be described explicitly our theorem gives presentations of $H^{*}(C(\mathcal{A}))$. We consider two such classes; the first one where $X$ is a geometric lattice and the other one where $C(\mathcal{A})$ is a so-called $k$ equal manifold. In the former case, the local homology groups of $X$ were computed in 7 13]; in the latter case, they were computed in [3]. We give presentations of the algebra $H^{*}(C(\mathcal{A}), \mathbb{Q})$ in these cases that in particular solves a problem stated in [3]. The representation for the former case was obtained independently and by a completely different method by E. M. Feichtner in [6].

The method we use in this paper is as follows. We start with the De ConciniProcesi differential graded algebra $M$ that is a specialization of the Morgan rational model of the complement of a divisor with normal crossings (see [10]). The algebra $M$ is a rational model of $C(\mathcal{A})$. We find inside $M$ a significantly smaller subalgebra $C M$ quasi-isomorphic to $M$ whence also a rational model of $C(\mathcal{A})$ (that may be of interest by itself). The algebra $C M$ gives a multiplicative structure on the flag complexes of $X$ that induces the ring structure on $\left.H^{*}(C(\mathcal{A})), \mathbb{Q}\right)$ described above. Moreover, $C M$ has a natural integral version $C M(\mathbb{Z})$ that gives the right groups $H^{p}(C(\mathcal{A})$ ) and is a good candidate for an integral model of $C(\mathcal{A})$ (see Remark 5.4 and Conjecture 6.7) 1

The paper is organized as follows. In section 2, we recall the De Concini-Procesi model $M$ and a monomial basis of it found in [15]. In section [3, we switch to generators more convenient for the purpose of the paper and exhibit a basis of monomials in the new generators. In section 4 we define the smaller subcomplex $C M$ that is a deformation retract of $M$. In section 5 we prove that $C M$ is a subalgebra of $M$ and study the multiplicative structure on it. In section [6, we describe the multiplication induced on its cohomology. In section 7 and 8 , we give a presentation of the algebra $H^{*}(C(\mathcal{A}))$ for the cases where $X$ is a geometric lattice and where $C(\mathcal{A})$ is a $k$-equal manifold.

This work was started when I was visiting Technische Universität in Berlin. I want to express my gratitude to G. Ziegler for his hospitality. I am also grateful to C. De Concini for useful communications and to Eva Feichtner for finding an error in the first version of Proposition 7.2

\section{De Concini-Procesi model}

In this section, we recall the construction of rational models from [5].

To follow closer the notation in [5] we dualize our initial definitions from the Introduction. Let $V$ be a finite dimensional complex linear space and $\mathcal{A}$ a finite set of nonzero linear subspaces of $V$. Without any loss of generality we can assume that their sum is $V$. The topological space we are interested in is $C(\mathcal{A})=V^{*} \backslash \bigcup_{A \in \mathcal{A}} A^{\circ}$ where $A^{\circ}$ is the annihilator of $A$ in $V^{*}$. The space $C(\mathcal{A})$ does not change if we include all nonempty sums of elements of $\mathcal{A}$ in $\mathcal{A}$. Therefore we will be dealing with

\footnotetext{
${ }^{1}$ November, 2000. This paper was written at the end of 1996 and has since been circulating as a preprint. The Conjecture 6.7 has been taken up repeatedly and was recently proven independently by de Longueville and Schultz [17] by direct topological methods and by Deligne, Goresky, and MacPherson [18] via a sheaf-theoretic approach. Also author's note [19] has appeared where even smaller and more natural rational model for subspace complements is constructed, using results of this paper.
} 
the lattice $X=X(\mathcal{A})$ of all sums of elements of $\mathcal{A}$ ordered by inclusion and labeled by the dimensions of its elements. Notice that $C(X)=C(\mathcal{A})=V^{*} \backslash \bigcup_{A \in X, A \neq 0} A^{\circ}$. Let us emphasize that the only structure on $X$ used in this paper is that of a labeled lattice. We also denote by $\mathbf{P}(A)$ the projectivization of a linear space $A$ and put $\mathbf{P} C(\mathcal{A})=\mathbf{P} V^{*} \backslash \bigcup_{A \in X, A \neq 0} \mathbf{P}\left(A^{\circ}\right)$.

Now we describe two different graded algebras (DGA) $M^{*}(X)$ and $M(X)$. There are different (though quasi-isomorphic to each other) versions of these algebras depending on the choice of a building set of $X$. Recall that a subset $G$ of $X$ is a building set if every $A \in X$ is the direct sum of those elements in $G$ that are maximal among elements of $G$ contained in $A$. For the most part of the paper we take the whole $X$ as the building set. The only time a different building set is used is for $X^{\prime}$ in this section.

Let $G$ be a building set containing $V$. Start with the polynomial algebra $P$ over $\mathbb{Q}$ with the indeterminates $c_{A}$ corresponding to $A \in G$ and the exterior algebra $\Lambda$ over $\mathbb{Q}$ generated by $e_{A}$ corresponding to $A \in G \backslash\{V\}$. Consider the DGA $P \otimes \Lambda$ with the grading induced by $\operatorname{deg} c_{A}=2$ and $\operatorname{deg} e_{A}=1$ and the differential generated by $d\left(e_{A}\right)=c_{A}$. Now consider the elements

$$
r\left(X_{1}, X_{2}, B\right)=\prod_{A \in X_{1}} e_{A} \prod_{A \in X_{2}} e_{A}\left(\sum_{C \supseteq B} c_{C}\right)^{d(Y, B)}
$$

of $P \otimes \Lambda$ where $X_{1}, X_{2} \subseteq G \backslash\{V\}, Y=X_{1} \cup X_{2}, B \in G, B \supsetneq A$ for all $A \notin Y$ and $d(Y, B)=\operatorname{dim} B-\operatorname{dim}\left(\sum_{A \in Y} A\right)$. Notice that in order to define the first product and thus $r\left(X_{1}, X_{2}, B\right)$ precisely one needs to introduce a linear order on $X_{1}$. Otherwise these elements are defined only up to \pm 1 ; this suffices for the following definition. Let $J$ be the ideal generated by all the elements $r\left(X_{1}, X_{2}, B\right)$. Clearly it is homogeneous and invariant with respect to $d$. Thus $M^{*}(X)=(P \otimes \Lambda) / J$ is a DGA. DGA $M(X)$ is defined similarly with the only difference being that both $e_{A}$ and $c_{A}$ are indexed by all the elements of $G$ (including $V$ ). We will denote by $M^{*}(X)_{r}$ and $M(X)_{r}$ the homogeneous components of degree $r$ of the algebras.

As it is customary, we will keep the notation $c_{A}$ and $e_{A}$ for the images of these elements under factorization and the term "monomials" for the images of monomials in $c_{A}$ and $e_{A}$.

Theorem 2.1 ([5, Theorem 5.3]). There is a $\mathbb{Q}$-algebra isomorphism $H^{*}\left(M^{*}(X)\right)$ $\approx H^{*}(\mathbf{P} C(\mathcal{A}), \mathbb{Q})$.

In [5, the problem of the naturality of this isomorphism is left open. It follows from [10] that after tensoring with $\mathbb{C}$ this isomorphism becomes canonical. Over $\mathbb{Q}$ it it not functorial in the category of smooth algebraic varieties ([10]). It is probably functorial in the category of arrangement complements but a proof is not known to me.

Now we want a model for $C(\mathcal{A}) \subset V^{*}$. We can view $\mathcal{A}$ as a subspace arrangement in $V^{\prime}=V \oplus W$ with $\operatorname{dim} W=1$ (this is a generalization of the coning construction from [13], p. 14). Then we can consider the new arrangement $\mathcal{A}^{\prime}=\mathcal{A} \cup\{W\} \cup$ $\{A \oplus W \mid A \in \mathcal{A}\}$ in $V^{\prime}$. Notice that $\mathbf{P} C\left(\mathcal{A}^{\prime}\right)=C(\mathcal{A})$. Thus $M^{*}\left(X^{\prime}\right)$ can serve as a model of $C(\mathcal{A})$.

To simplify definition of $M^{*}\left(X^{\prime}\right)$ we choose $X \cup\left\{W, V^{\prime}\right\}$ as the building set for $X^{\prime}$. Then to obtain $M^{*}\left(X^{\prime}\right)$ one needs to adjoin $e_{W}, e_{V}, c_{V^{\prime}}$ and $c_{W}$ to the 
generators of $M^{*}(X)$. Taking $X_{1}=X_{2}=\emptyset$ and $B=W$, we obtain the relation

$$
c_{W}+c_{V^{\prime}}=0 .
$$

Thus $c_{W}=-c_{V^{\prime}}$ and we can forget about $c_{W}$.

The next observation is that we can substitute simpler DGA $M(X)$ for $M^{*}\left(X^{\prime}\right)$.

Proposition 2.2. The assignment $e_{A} \mapsto e_{A}(A \in X \backslash\{V\}), c_{B} \mapsto c_{B}(B \in X)$, $e_{W} \mapsto 0$, and $c_{V^{\prime}} \mapsto 0$ generates a surjective DGA morphism $\phi: M^{*}\left(X^{\prime}\right) \rightarrow M(X)$ that induces an isomorphism in cohomology.

Proof. A direct check shows that the assignment from the statement sends every relation for $M^{*}\left(X^{\prime}\right)$ to a relation for $M(X)$. Thus it generates an algebra homomorphism $\phi$. Clearly $\phi$ preserves the gradings, commutes with the differentials, and is surjective.

The kernel of $\phi$ is the ideal $I$ of $M^{*}\left(X^{\prime}\right)$ generated by $e=e_{W}$ and $c=c_{V^{\prime}}$. Using the relations for this algebra it is easy to exhibit a linear basis of $I$ : $\left\{e c^{k}, c^{k+1}, a_{A} c^{m}, c_{A} c^{m}\right\}$ where $0 \leq k<\operatorname{dim} V^{\prime}-1, A \in X$, and for each $A$ we have $1 \leq m<\operatorname{dim} V^{\prime}-\operatorname{dim} A$ (cf. Proposition 2.3). This basis is invariant for $d$ up to \pm 1 ; more exactly $d\left(e c^{k}\right)=-c^{k+1}$ and $d\left(e_{A} c^{m}\right)=c_{A} c^{m}$. This shows that $I$ is an acyclic cochain complex and the last claim of the proposition follows from the exact cohomology sequence of the exact sequence

$$
0 \rightarrow I \rightarrow M^{*}\left(X^{\prime}\right) \rightarrow M(X) \rightarrow 0
$$

Proposition 2.2 implies that $M(X)$ can be taken as a model of $C(\mathcal{A})$. Keeping this in mind we will focus our attention on $M=M(X)$ from now on. Let us emphasize again that the building set for $M$ is the whole $X$.

Our goal for the rest of the section is to exhibit a linear basis of $M$ consisting of monomials in the generators $c_{A}$ and $e_{A}$. For each $T \subseteq X$ denote by $\mu(T)$ the monomial of $\Lambda$ corresponding to $T$ (defined up to \pm ). Then consider the grading $\Lambda=\bigoplus_{T \subseteq X} \Lambda^{T}$ of $\Lambda$ where $\Lambda^{T}=\mathbb{Q} \mu(T)$. It generates a grading on $\Lambda \otimes P$ and, since every $r\left(X_{1}, X_{2}, B\right)$ is homogeneous in this grading, a grading $M=\bigoplus_{T \subset X} M^{T}$ on $M$. Then notice that for every $A_{1}, A_{2} \in X$ neither of which is a subset of the other

$$
a_{A_{1}} a_{A_{2}}=0
$$

in $M$ where $a_{A}$ denotes either $c_{A}$ or $e_{A}$. To see this it is enough to take in (2.1) $Y=\left\{A_{1}, A_{2}\right\}$ and $B=A_{1}+A_{2}$ that gives $d(Y, B)=0$. Hence all the monomials in $c_{A}$ identified up to the ordering (or \pm 1 ) are in one-to-one correspondence with flags (by inclusion) in $X$. In particular, in the representation $M=\bigoplus_{T \subset X} M^{T}$, a summand $M^{T}$ is nonzero only if $T$ is a flag in $X$. In any case $M^{T}$ has the following structure of a graded algebra (although not a subalgebra of $M$ ). Denote by $X^{T}$ the set of all elements of $X$ each one of which forms a flag with $T$. Then $M^{T}$ is the factor of the polynomial ring $\mathbb{Q}\left[c_{A} \mid A \in X^{T}\right]$ by the ideal generated by

$$
r(Y, B)=\prod_{A \in Y} c_{A}\left(\sum_{C \supseteq B} c_{C}\right)^{d^{T}(Y, B)}
$$

for every $Y \subset X^{T} \backslash T, B \in X^{T}$ such that $B \supsetneq A$ for every $A \in Y$, and $d^{T}(Y, B)=$ $\operatorname{dim} B-\operatorname{dim}\left(\sum_{A \in Y \cup T, A \subset B} A\right)$. (In fact in [5] the algebras $M^{T}$ appeared first and $M^{*}(X)$ was constructed from them following [10].) 
A monomial basis of algebra $M^{T}$ was first constructed in [15] (see 15], Remark 3.11) and then generalized in [8]. Combining the monomial bases of $M^{T}$ for all flags $T$ we obtain the following.

Proposition 2.3. Suppose $S, T \subseteq X$ (not necessarily disjoint), $T=\left\{A_{1}, \ldots, A_{k}\right\}$ is ordered by inclusion and $S \cup T$ is a flag. Suppose $m: S \rightarrow \mathbf{Z}_{+}$satisfies $m(A)<$ $\operatorname{dim} A-\operatorname{dim} A^{\prime}$ for every $A \in S$ where $A^{\prime}=0$ if $A$ is the smallest element of $T \cup S$ and $A^{\prime}$ is the predecessor of $A$ in $T \cup S$ otherwise. Then the monomials $\lambda(S, T, m)=e_{A_{1}} \cdots e_{A_{k}} \prod_{B \in S} c_{B}^{m(B)}$ form a basis of $M$.

We will need later one more idea from [15. Consider an arbitrary monomial $\lambda=e_{A_{1}} \cdots e_{A_{k}} c_{B_{1}}^{m_{1}} \cdots c_{B_{l}}^{m_{l}}$. Then define the weight of $\lambda$ as

$$
\operatorname{wt}(\lambda)=\sum_{i=1}^{k} \operatorname{dim} A_{i}+2 \sum_{i=1}^{l} m_{k} \operatorname{dim} B_{i} .
$$

It is easy to see that for any monomial $\lambda(S, T, m)$ that appears in the linear decomposition of $\lambda$ with a nonzero coefficient we have

$$
\operatorname{wt}(\lambda(S, T, m)) \geq \operatorname{wt}(\lambda)
$$

(cf. [15], proof of Proposition 2.2).

\section{NEW GENERATORS}

In this section, we switch to new generators of algebra $M$. For every $A \in X$ put

$$
\sigma_{A}=\sum_{B \supseteq A} c_{B} \quad \text { and } \quad \tau_{A}=\sum_{B \supseteq A} e_{B} .
$$

Clearly $\left\{\sigma_{A}, \tau_{A}\right\}$ is a generating set of $M$.

The new generators satisfy many relations that follow from the relations for $c_{A}$ and $e_{A}$. We notice some of them for future use. We use the symbol $\rho_{A}$ meaning either $\sigma_{A}$ or $\tau_{A}$.

(3.1). All $\sigma_{A}$ have degree 2 and thus belong to the center of $M$. The elements $\tau_{A}$ are degree 1 generators of an exterior subalgebra of $M$. In particular $\tau_{A}^{2}=0$ and $\tau_{A} \tau_{B}=-\tau_{B} \tau_{A}$. Thus all monomials in $\tau_{A}$ and $\sigma_{A}$ can be parametrized by the triples $(S, T, m)$ where $S, T \subseteq X$, a linear ordering on $T$ is fixed and $m: S \rightarrow \mathbf{Z}_{+}$. We put

$$
\mu(S, T, m)=\prod_{B \in T} \tau_{B} \prod_{A \in S} \sigma_{A}^{m(A)}
$$

where the first product is taken according to the order on $T$. Note that $\operatorname{deg} \mu(S, T, m)$ $=|T|+2 \sum_{A \in S} m(A)$.

(3.2). For every $A, B \in X$ we have

$$
\tau_{A} \tau_{B}=\tau_{A} \tau_{A+B}-\tau_{B} \tau_{A+B}
$$

Proof. Represent $\tau_{A}=\Delta_{A, B}+\tau_{A+B}$ where

$$
\Delta_{A, B}=\sum_{C \supseteq A, C \nsupseteq B} e_{C} .
$$


Similarly $\tau_{B}=\Delta_{B, A}+\tau_{A+B}$. Since for every $C_{1} \supseteq A, C_{1} \nsupseteq B$ and $C_{2} \supseteq B, C_{2} \nsupseteq A$ the set $\left\{C_{1}, C_{2}\right\}$ is not a flag we have $e_{C_{1}} e_{C_{2}}=0$ whence $\Delta_{A, B} \Delta_{B, A}=0$. Using besides that $\tau_{A+B}^{2}=0$ we obtain the result.

(3.3). For every $A \in X$ we have

$$
\sigma_{A}^{\operatorname{dim} A}=0
$$

and

$$
\rho_{A} \sigma_{B}^{k}=\rho_{B} \sigma_{B}^{k}
$$

for every $A \subseteq B$ and $k \geq \operatorname{dim} B-\operatorname{dim} A$.

Proof. The first relation follows immediately from (2.1) if one takes $Y=\emptyset$ and $B=A$. In order to prove the second one it suffices to take $k=\operatorname{dim} B-\operatorname{dim} A$. Fix $A, B \in X$ such that $A \subset B$ and put $k=\operatorname{dim} B-\operatorname{dim} A$. We have

$$
\rho_{A} \sigma_{B}^{k}=\sum_{C \supseteq A} a_{C} \sigma_{B}^{k}=\sum_{C \supseteq A} a_{C}\left(\sum_{D \supseteq B} c_{D}\right)^{k}
$$

where $a_{A}=c_{A}$ or $e_{A}$ respectively for $\rho_{A}=\sigma_{A}$ or $\tau_{A}$. Consider the sum $b_{C}=$ $a_{C}\left(\sum_{D \supseteq B} c_{D}\right)^{k}$ for some $C$ such that $C \supseteq A$ but $C \nsupseteq B$. If there is no inclusion between $C$ and $D$, then $a_{C} c_{D}=0$. So it suffices to sum over $D$ such that $D \supseteq C$, i.e. $D \supseteq B+C$. In other words,

$$
b_{C}=a_{C}\left(\sum_{D \supseteq B+C} c_{D}\right)^{k} .
$$

Now we have

$$
\operatorname{dim}(B+C)-\operatorname{dim} C=\operatorname{dim} B-\operatorname{dim} B \cap C \leq \operatorname{dim} B-\operatorname{dim} A=k .
$$

Due to (2.1) $b_{C}=0$ and the summation in (3.4) can be taken over $C$ such that $C \supseteq B$.

Now we are ready to exhibit a basis of $M$ consisting of monomials in $\sigma_{A}$ and $\tau_{A}$.

Proposition 3.1. Suppose that $S, T \subseteq X, S \cup T$ is a flag (by inclusion), the ordering on $T$ coincides with the inclusion order, $m(A)<\operatorname{dim} A-\operatorname{dim} A^{\prime}$ for every $A \in S$ and $A^{\prime}$ as in Proposition 2.3, and finally $|T|+2 \sum_{A \in S} m(A)=r$ for a fixed nonnegative $r$. Then the set of monomials $\mu(S, T, m)$ is a linear basis of $M_{r}$ (we call the monomials in this set basic monomials).

Proof. Comparing the set of basic monomials of degree $r$ with the basis of $M_{r}$ from section 2 we see that they have the same number of elements. Thus it suffices to prove that every monomial $\lambda=\lambda(S, T, m)$ in $c_{A}$ and $e_{A}$ with $m(A)<\operatorname{dim} A-\operatorname{dim} A^{\prime}$ and $2 \sum_{A \in S} m(A)+|T|=r$ is a linear combination of basic monomials of degree $r$. Apply downward induction on weight of $\lambda$. Clearly the weight of any monomial of degree $r$ is limited from above by $n r$ where $n=\operatorname{dim} V$ and the monomial of weight $n r$ is either $c_{V}^{r / 2}=\sigma_{V}^{r / 2}$ if $r$ is even or $e_{V} c_{V}^{(r-1) / 2}=\tau_{V} \sigma_{V}^{(r-1) / 2}$ otherwise. Since this monomial is 0 if $\lfloor r / 2\rfloor \geq n$ (cf. [3.3) and basic otherwise we have the base of the induction.

Let $\lambda=\lambda(S, T, m)$ be an arbitrary monomial in $c_{A}$ and $e_{A}$ of degree $r$ with $m(A)<\operatorname{dim} A-\operatorname{dim} A^{\prime}$ and $\operatorname{wt}(\lambda)<n r$. Consider the polynomial $p=\lambda-\mu(S, T, m)$ 
in $c_{A}$ and $e_{A}$. Clearly every monomial of $p$ has a weight larger than the weight of $\lambda$ whence the same is true for each monomial of the decomposition of $p$ into the basis of section 2 (compare the remarks on weights after Proposition 2.3). Thus the induction works.

\section{CRitical monomials}

Whenever we consider a monomial $\mu(S, T, m)$ in the rest of the paper, if $S \subset T$ we extend $m$ to $T$ by 0 .

Definition 4.1. A basic monomial $\mu(S, T, m)$ is critical if $T \supseteq S$ and $m(A)=$ $\operatorname{dim} A-\operatorname{dim} A^{\prime}-1$ for every $A \in T$ (so $S=\left\{A \in T \mid \operatorname{dim} A-\operatorname{dim} A^{\prime}>1\right\}$ ).

Notice that the set of all critical monomials is linearly independent in $M$. Notice also that a critical monomial is defined by a flag $T$ provided with the dimensions of its elements. Denote by $c \mu(T)$ the critical monomial defined by $T$. We have

$$
\operatorname{deg} c \mu(T)=2 \operatorname{dim} A(T)-|T|
$$

where $A(T)$ is the maximal element of $T$. Denote by $C M$ the linear subspace of $M$ spanned by all the critical monomials.

Proposition 4.2. Let $T=\left\{A_{1}, A_{2}, \ldots, A_{k}\right\}$ where $A_{i} \subset A_{i+1}$ and let $T_{i}=\left\{A_{1}, \ldots\right.$, $\left.\hat{A}_{i}, \ldots, A_{k}\right\}$ for every $i=1,2, \ldots, k$. Then

$$
d(c \mu(T))=\sum_{i=1}^{k-1}(-1)^{i} c \mu\left(T_{i}\right) .
$$

Proof. To simplify notation put $\rho_{i}=\rho_{A_{i}}$ and $m(i)=\operatorname{dim} A_{i}-\operatorname{dim} A_{i-1}-1$. Using the Leibniz formula for $d$ and the relations (3.3) repeatedly we have

$$
\begin{aligned}
& d(c \mu(T))=d\left(\tau_{1} \cdots \tau_{k} \sigma_{1}^{m(1)} \cdots \sigma_{k}^{m(k)}\right) \\
& \quad=\sum_{i=1}^{k}(-1)^{i-1} \tau_{1} \cdots \hat{\tau}_{i} \cdots \tau_{k} \sigma_{1}^{m(1)} \cdots \sigma_{i}^{m(i)+1} \cdots \sigma_{k}^{m(k)} \\
& =\sum_{i=2}^{k}(-1)^{i-1} \tau_{1} \cdots \widehat{\tau_{i-1}} \cdots \tau_{k} \sigma_{1}^{m(1)} \cdots \widehat{\sigma_{i-1}^{m(i-1)}} \sigma_{i}^{m(i)+m(i-1)+1} \cdots \sigma_{k}^{m(k)} \\
& =\sum_{i=1}^{k-1}(-1)^{i} c \mu\left(T_{i}\right)
\end{aligned}
$$

where the third equality follows from

$$
\tau_{i-1} \sigma_{i-1}^{m(i-1)} \sigma_{i}^{m(i)+1}=\tau_{i} \sigma_{i}^{m(i-1)+m(i)+1}
$$

for $i \geq 2$ by the second relation in $(3.3)$ and

$$
\sigma_{1}^{m(1)+1}=0
$$

by the first relation in $(3.3)$

Corollary 4.3. The linear space $C M$ is a subcomplex of $M$. 
Remark 4.4. Proposition 4.2 implies that the complex $C M$ affords the grading $C M=\bigoplus_{A \in X} C M_{A}$ where $C M_{A}$ is generated by monomials $c \mu(T)$ with $A(T)=A$. The deletion of $A$ from all the flags gives an isomorphism of degree -1 from $C M_{A}$ to the usual flag complex $F(A)$ of the poset $(0, A)$. This relates the De Concini-Procesi model for $C(\mathcal{A})$ and the Goresky-MacPherson formula via

$$
H^{p}(C M)=\bigoplus_{A \in X} H_{2 \operatorname{dim} A-p-2}(F(A))
$$

(see section 6).

Our next goal is to prove that $C M$ is quasi-isomorphic to $M$. Denote by $C M^{\perp}$ the linear space spanned by all the basic monomials that are not critical. Clearly $M=C M \oplus C M^{\perp}$ as a linear space. The next lemma proves that it is true in the category of complexes also.

Lemma 4.5. The space $C M^{\perp}$ is a subcomplex of $M$.

Proof. Let $\mu=\mu(S, T, m) \in C M^{\perp}$. This means that $\mu$ satisfies one of the following conditions.

1. $S \nsubseteq \nsubseteq T$, i.e., there exists $A \in X$ such that $\sigma_{A}$ divides $\mu$ and $\tau_{A}$ does not. Then every monomial in $d(\mu)$ satisfies the same condition whence $d(\mu) \in C M^{\perp}$.

2. $S \subseteq T$ and there exists $A \in T$ such that $m(A)<\operatorname{dim} A-\operatorname{dim} A^{\prime}-1$. Suppose $T=\left\{A_{1}, \ldots, A_{k}\right\}$ and $A=A_{i}$. As in the proof of Proposition 4.2 we put $\rho_{A_{j}}=\rho_{j}$, $m\left(A_{j}\right)=m(j)$ and have

$$
d(\mu)=\sum_{j=1}^{k}(-1)^{j-1} \mu_{j}
$$

where

$$
\mu_{j}=\tau_{1} \cdots \hat{\tau}_{j} \cdots \tau_{k} \sigma_{1}^{m(1)} \cdots \sigma_{j}^{m(j)+1} \cdots \sigma_{k}^{m(i)} .
$$

Clearly, $\mu_{j}=\mu\left(T_{j}, S^{j}, m^{(j)}\right)$ for some $S^{j}$ and a function $m^{(j)}$.

Consider several cases. If $m(j)<\operatorname{dim} A_{j}-\operatorname{dim} A_{j-1}-1$ then $\mu_{j}$ is basic and $S^{j} \nsubseteq T_{j}$ whence $\mu_{j} \in C M^{\perp}$. Suppose $m(j)=\operatorname{dim} A_{j}-\operatorname{dim} A_{j-1}-1$, in particular $j \neq i$. For $j=1$, the exponent of $\sigma$ in $\mu_{1}$ is $\operatorname{dim} A_{1}-1$, whence $\mu_{1}=0$ by the first relation in (3.3). For $j>1$, using the same computation as in the proof of Proposition 4.2 we have

$$
\mu_{j}=\tau_{1} \cdots \hat{\tau}_{j-1} \cdots \tau_{k} \sigma_{1}^{m(1)} \cdots \sigma_{j-1}^{\widehat{m(j-1)}} \sigma_{j}^{m(j)+m(j-1)+1} \cdots \sigma_{k}^{m(k)} .
$$

The monomial $\mu_{j}$ is again basic. If $i \neq j-1$, then the exponent of $\sigma_{i}$ in $\mu_{j}$ is $m(i)$ whence $\mu_{j} \in C M^{\perp}$. Finally if $i=j-1$, then the exponent of $\sigma_{j}$ in $\mu_{j}$ is

$$
\begin{aligned}
m(j) & +m(i)+1<\operatorname{dim} A_{j}-\operatorname{dim} A_{i}-1+\operatorname{dim} A_{i}-\operatorname{dim} A_{i-1}-1+1 \\
& =\operatorname{dim} A_{j}-\operatorname{dim} A_{j-2}-1
\end{aligned}
$$

whence again $\mu_{j} \in C M^{\perp}$. That completes the proof.

Now we are to construct a homotopy on $C M^{\perp}$ between the identity map and 0 .

For every basic monomial $\mu=\mu(S, T, m)$ and $A \in S \backslash T$ put $h_{A} \mu=$ $\mu(S, T \cup\{A\}, m) / \sigma_{A}$. Then put $h \mu=\sum_{A \in S \backslash T}(-1)^{(A, T)} h_{A} \mu$ where $(A, T)=$ $|\{B \in T \mid B \subset A\}|$. Also for every $B \in T$ put $d^{B}(\mu)=\mu(S, T \backslash\{B\}, m) \sigma_{B}$. Notice that $d(\mu)=\sum_{B \in T}(-1)^{(B, T)} d^{B}(\mu)$ where $(B, T)=|\{C \in T \mid C \subset B\}|$. Call $B \in T$ 
critical for $\mu$ if $m(B)=\operatorname{dim} B-\operatorname{dim} B^{\prime}-1$ (as usual put $m(B)=0$ if $\left.B \notin S\right)$. Denote by $C T$ the set of all critical elements of $T$. Now put $|\mu(S, T, m)|=|S \cup T \backslash C T|$. Notice that $|\mu| \neq 0$ if and only if $\mu \in C M^{\perp}$.

Proposition 4.6. The linear map $C M^{\perp} \rightarrow C M^{\perp}$ defined by $\mu \mapsto(1 /|\mu|) h \mu$ is a homotopy between the identity map and 0 .

Proof. It suffices to check that for every basic monomial $\mu$ we have

$$
h d(\mu)+d(h \mu)=|\mu| \mu .
$$

To make the check easier we state several simple observations. In them and in the rest of the proof we always mean that $\mu=\mu(S, T, m)$. Also all the equalities mean in particular that the expressions in them are defined.

(a) For every $A \in S \backslash T$ and $B \in T \backslash C T$ we have $h_{A} d^{B}(\mu)=d^{B}\left(h_{A} \mu\right)$.

(b) For every $A$ and $B$ as in (a) we have $d^{A}\left(h_{A} \mu\right)=\mu=h_{B} d^{B}(\mu)$.

(c) For every $A \in S \backslash T$ the set of all critical for $h_{A} \mu$ elements coincides with $C T$.

(d) For $B \in C T$, let $\mu\left(S^{\prime}, T^{\prime}, m^{\prime}\right)$ be the basic monomial equal to $d^{B}(\mu)$.

(d.1) If $B^{\prime} \in T$, then $S^{\prime} \backslash T^{\prime}=S \backslash T$.

(d.2) If $B^{\prime} \notin T$, then $S^{\prime} \backslash T^{\prime}=(S \backslash T \cup\{B\}) \backslash\left\{B^{\prime}\right\}$.

(e) Let again $B \in C T$ and $A \in S \backslash T$.

(e.1) If $B^{\prime} \in T$ or $A \neq B^{\prime}$, then $h_{A} d^{B}(\mu)=d^{B}\left(h_{A} \mu\right)$.

(e.2) If $B \notin T$, then $h_{B} d^{B}(\mu)=-d^{B} h_{B^{\prime}}(\mu)$ and $d^{B^{\prime}} h_{B^{\prime}}(\mu)=\mu$.

The statements (a)-(c) are straightforward. To check (d) and (e) apply relations of type (3.3) several times.

Combining (a)-(e) and using the rule for signs in the Leibniz property of $d$ one gets (4.1).

Corollary 4.7. The embedding $C M \subset M$ is a quasi-isomorphism of the complexes.

This corollary together with Remark 4.4 imply the Goresky-MacPherson formula for the cohomology groups of $C(\mathcal{A})$.

\section{Multiplicative structure of $C M$}

In this section, we prove that $C M$ is a subalgebra of $M$ and compute the product of two critical monomials explicitly in terms of the poset $X$ labeled by dimension.

In fact, we do first a more general computation. Consider the $\mathbb{C}$-linear space $W$ of all formal linear combinations of the pairs $(T, m)$ where $T \subseteq X$ is a flag and $m: T \rightarrow \mathbf{N}$. We want to convert $W$ into an associative algebra. For that we recall the shuffle product on the free Abelian group generated by flags (cf. [13], 3.4). Let $T_{1}=\left(A_{1} \subset A_{2} \subset \cdots \subset A_{p}\right)$ and $T_{2}=\left(B_{1} \subset B_{2} \subset \cdots \subset B_{q}\right)$ and $m_{i}: T_{i} \rightarrow \mathbf{N}(i=1,2)$. Denote by $\lambda$ the natural operator that turns an arbitrarily subset of $X$ into a flag, where the subset is equipped with a linear order independent of the order on the poset. More precisely, for $\left\{C_{1}, \ldots, C_{k}\right\} \subseteq X$, linearly ordered along increasing indexes

$$
\lambda\left(C_{1}, C_{2}, \ldots, C_{k}\right)=\left(C_{1}, C_{1}+C_{2}, \ldots, C_{1}+C_{2}+\cdots+C_{k}\right)
$$

if all the elements in the right-hand side are distinct and $\lambda\left(C_{1}, C_{2}, \ldots, C_{k}\right)=0$ otherwise. Then define

$$
T_{1} \circ T_{2}=\sum_{\pi}(\operatorname{sign} \pi) \lambda \pi\left(T_{1} \cup T_{2}\right)
$$


where $\pi$ runs through all $(p, q)$-shuffles of $T_{1} \cup T_{2}$ (these are the permutations preserving the order among $A_{i}$ 's and the order among $B_{j}$ 's). It is easy to check that the defined multiplication is associative (see [13], p. 88). For the future use put for a $(p, q)$-shuffle $\pi$ of $T_{1} \cup T_{2}$

$$
\left(T_{1} \cup T_{2}\right)^{\pi}=\lambda \pi\left(T_{1} \cup T_{2}\right) .
$$

We want to extend this multiplication to $W$. For that fix a $(p, q)$-shuffle $\pi$ and notice that the flag $\left(T_{1} \cup T_{2}\right)^{\pi}$ consists of some of the spaces $A_{i}+B_{j}$ where $0 \leq i \leq p$ and $0 \leq j \leq q$ (we always put $A_{0}=B_{0}=0$ ). Notice also that the predecessor of $A_{i}+B_{j}$ is either $A_{i-1}+B_{j}$ or $A_{i}+B_{j-1}$ (where the predecessor of the smallest element $A_{1}=A_{1}+B_{0}$ (or $\left.B_{1}=A_{0}+B_{1}\right)$ is taken to be $\left.A_{0}+B_{0}=0\right)$. Define $\left(m_{1} \circ m_{2}\right)^{\pi}:\left(T_{1} \cup T_{2}\right)^{\pi} \rightarrow \mathbf{N}$ via

$$
\left(m_{1} \circ m_{2}\right)^{\pi}\left(A_{i}+B_{j}\right)=m_{1}\left(A_{i}\right) \quad \text { or } \quad m_{2}\left(B_{j}\right)
$$

if the predecessor of $A_{i}+B_{j}$ is $A_{i-1}+B_{j}$ or $A_{i}+B_{j-1}$ respectively. Now put

$$
\left(T_{1}, m_{1}\right) \circ\left(T_{2}, m_{2}\right)=\sum_{\pi} \operatorname{sign}(\pi)\left(\left(T_{1} \cup T_{2}\right)^{\pi},\left(m_{1} \circ m_{2}\right)^{\pi}\right)
$$

where $\pi$ again runs through the group of $(p, q)$-shuffles of $T_{1} \cup T_{2}$. The associativity of this product follows easily from the associativity of the shuffle product of flags. Hence this product defines a structure of an associative algebra on $W$ (with the identity represented by the empty flag).

Define the linear map $f: W \rightarrow M$ via $f(\emptyset)=1$ and

$$
f((T, m))=\tau_{A_{1}} \cdots \tau_{A_{p}} \prod_{i=1}^{p} \sigma_{A_{i}}^{m\left(A_{i}\right)}
$$

for $T=\left(A_{1} \subset \cdots \subset A_{p}\right)$.

The main theorem of this section is as follows.

Theorem 5.1. The map $f$ is a homomorphism of the algebras.

Proof. Fix $\left(T_{1}, m_{1}\right)$ and $\left(T_{2}, m_{2}\right)$ from $W$ where $T_{1}=\left(A_{1} \subset \cdots \subset A_{p}\right)$ and $T_{2}=$ $\left(B_{1} \subset \cdots \subset B_{q}\right)$. We need to prove that

$$
f\left(T_{1}, m_{1}\right) f\left(T_{2}, m_{2}\right)=f\left(\left(T_{1}, m_{1}\right) \circ\left(T_{2}, m_{2}\right)\right) .
$$

First we consider particular cases.

(a) Suppose $m_{i}=0(i=1,2)$ and $p=q=1$. Then (5.1) is a relation of type

(b) Suppose $m_{i}=0(i=1,2)$ and $p=1$. Putting $A=A_{1}$ we have (5.1) in the form

$$
\tau_{A} \tau_{B_{1}} \cdots \tau_{B_{q}}=\sum_{r=0}^{q}(-1)^{r} \tau_{B_{1}} \cdots \tau_{B_{r}} \tau_{A+B_{r}} \cdots \tau_{A+B_{q}} .
$$

We prove (5.2) by induction on $q$ using (a) as the base for $q=1$. For $q>1$ by the inductive hypothesis we have

$$
\tau_{A} \tau_{B_{1}} \cdots \tau_{B_{q}}=\sum_{r=0}^{q-1}(-1)^{r} \tau_{B_{1}} \cdots \tau_{B_{r}} \tau_{A+B_{r}} \cdots \tau_{A+B_{q-1}} \tau_{B_{q}} .
$$


Applying relation $(3.2)$ to the last two factors of (5.3) we can substitute instead of them

$$
\tau_{A+B_{q-1}} \tau_{A+B_{q}}-\tau_{B_{q}} \tau_{A+B_{q}}
$$

From the second summand of (5.4) we get $q-1$ monomials ending with

$$
\tau_{A+B_{q-2}} \tau_{B_{q}} \tau_{A+B_{q}}
$$

and the monomial

$$
\tau_{B_{1}} \cdots \tau_{B_{q-1}} \tau_{B_{q}} \tau_{A+B_{q}}
$$

with coefficient $(-1)^{q}$. Applying again relation (3.2) to the first two factors from (5.5) and using that $A+B_{q-2}+B_{q}=A+B_{q}$ and $\tau_{A+B_{q}}^{2}=0$ we obtain that all $q-1$ monomials of the first kind are 0. Thus (5.2) follows.

(c) Suppose $m_{i}=0(i=1,2)$. The proof is straightforward by induction on $p$ using (b) as the base for $p=1$ and the associativity of the shuffle product.

(d) To finish the proof we need the following claim:

$$
\tau_{A} \tau_{A+B} \sigma_{B}=\tau_{A} \tau_{A+B} \sigma_{A+B}
$$

for every $A, B \in X$. The proof is similar to the proof of relation (3.2) using also that $\tau_{A+B}^{2}=0$. The details are left to the reader.

(e) Consider the general case. By (c) the left-hand side of (5.1) can be rewritten as

$$
\sum_{\pi}(\operatorname{sign} \pi) f\left(\left(T_{1} \cup T_{2}\right)^{\pi}, 0\right) \prod_{i=1}^{p} \sigma_{A_{i}}^{m_{1}\left(A_{i}\right)} \prod_{j=1}^{q} \sigma_{B_{j}}^{m_{2}\left(B_{j}\right)} .
$$

Fix a $\pi$ and denote the respective monomial of (5.7) by $s(\pi)$. Suppose $A_{i}+B_{j} \in$ $\left(T_{1} \cup T_{2}\right)^{\pi}$. If $A_{i-1}+B_{j} \in\left(T_{1} \cup T_{2}\right)^{\pi}$ (and thus precedes $A_{i}+B_{j}$ ) then apply (d) to

$$
\tau_{A_{i-1}+B_{j}} \tau_{A_{i}+B_{j}} \sigma_{A_{i}}^{m_{1}\left(A_{i}\right)}
$$

$m_{1}\left(A_{i}\right)$ times. Since $A_{i-1}+B_{j}+A_{i}=A_{i}+B_{j}$ we can substitute

$$
\tau_{A_{i-1}+B_{j}} \tau_{A_{i}+B_{j}} \sigma_{A_{i}+B_{j}}^{m_{1}\left(A_{i}\right)}=\tau_{A_{i-1}+B_{j}} \tau_{A_{i}+B_{j}} \sigma_{A_{i}+B_{j}}^{\left(m_{1} \circ m_{2}\right)^{\pi}\left(A_{i}+B_{j}\right)}
$$

instead of (5.8). The case where $A_{i}+B_{j-1} \in\left(T_{1} \cup T_{2}\right)^{\pi}$ can be handled similarly. Since eventually every factor of the form $\sigma_{C}^{k}$ is used we obtain

$$
s(\pi)=f\left(\left(T_{1} \cup T_{2}\right)^{\pi},\left(m_{1} \circ m_{2}\right)^{\pi}\right)
$$

which completes the proof.

Corollary 5.2. Recall that $c \mu(T)$ denotes the critical monomial defined by $T$ (compare the beginning of Section 4$)$. For every two flags $T_{1}$ and $T_{2}$ with the maximal elements $A$ and $B$ respectively we have

$$
c \mu\left(T_{1}\right) c \mu\left(T_{2}\right)=\sum_{\pi}(\operatorname{sign} \pi) c \mu\left(T_{1} \cup T_{2}\right)^{\pi}
$$

if $A \cap B=0$ (equivalently $\operatorname{dim}(A+B)=\operatorname{dim} A+\operatorname{dim} B)$ and

$$
c \mu\left(T_{1}\right) c \mu\left(T_{2}\right)=0
$$

otherwise. Here again $\pi$ runs through all the $\left(\left|T_{1}\right|,\left|T_{2}\right|\right)$-shuffles of $T_{1} \cup T_{2}$. 
Proof. Fix $T_{1}$ and $T_{2}$ as in the proof of the theorem above and fix a $(p, q)$-shuffle $\pi$. Define $m_{k}: T_{k} \rightarrow \mathbb{Z}_{+}(k=1,2)$ via $m_{1}\left(A_{i}\right)=\operatorname{dim} A_{i}-\operatorname{dim} A_{i-1}-1$ and $m_{2}\left(B_{j}\right)=\operatorname{dim} B_{j}-\operatorname{dim} B_{j-1}-1$. By definition $c \mu\left(T_{k}\right)=f\left(\left(T_{k}, m_{k}\right)\right)(k=1,2)$.

Suppose first that $A_{p} \cap B_{q}=0$. We need to show that $\left(m_{1} \circ m_{2}\right)^{\pi}$ coincides with the exponent function in $c \mu\left(T_{1} \cup T_{2}\right)^{\pi}$. Let $A_{i}+B_{j} \in\left(T_{1} \cup T_{2}\right)^{\pi}$. If its predecessor is $A_{i-1}+B_{j}$, then we have

$$
\begin{gathered}
\left(m_{1} \circ m_{2}\right)^{\pi}\left(A_{i}+B_{j}\right)=m_{1}\left(A_{i}\right)=\operatorname{dim} A_{i}-\operatorname{dim} A_{i-1}-1 \\
=\operatorname{dim}\left(A_{i}+B_{j}\right)-\operatorname{dim}\left(A_{i-1}+B_{j}\right)-1
\end{gathered}
$$

since $A_{i} \cap B_{j}=A_{i-1} \cap B_{j}=0$. The case where the predecessor is $A_{i}+B_{j-1}$ can be handled similarly. Thus

$$
f\left(\left(T_{1} \cup T_{2}\right)^{\pi},\left(m_{1} \circ m_{2}\right)^{\pi}\right)=c \mu\left(\left(T_{1} \cup T_{2}\right)^{\pi}\right)
$$

which completes the proof of the first case.

Now suppose that $A_{p} \cap B_{q} \neq 0$. Then there exists $A_{i}+B_{j} \in\left(T_{1} \cup T_{2}\right)^{\pi}$ that is the smallest (by inclusion) element such that $\operatorname{dim}\left(A_{i}+B_{j}\right)<\operatorname{dim} A_{i}+\operatorname{dim} B_{j}$. To simplify notation put $m=\left(m_{1} \circ m_{2}\right)^{\pi}, T=\left(T_{1} \cup T_{2}\right)^{\pi}$ and denote all the elements of $T$ from the smallest to $A_{i}+B_{j}$ by $C_{1}, C_{2}, \ldots, C_{k}$. Then similar calculations as in (5.9) give $m\left(C_{i}\right)=\operatorname{dim} C_{i}-\operatorname{dim} C_{i-1}-1$ for $i<k$ and $m\left(C_{k}\right) \geq \operatorname{dim} C_{k}-\operatorname{dim} C_{k-1}$. Applying several times relation $(3.3)$ we see that $f((T, m))$ is equal to a monomial having $\sigma_{C_{k}}^{l}$ in it with $l \geq \operatorname{dim} C_{k}$. Thus $f((T, m))=0$, which completes the proof.

Now Corollaries 5.2 and 4.7 imply the following.

Corollary 5.3. $C M$ is a (differential graded) subalgebra of $M$ and a rational model of $C(\mathcal{A})$.

Remark 5.4. DGA $C M$ has a natural integer version (and a differential graded subring) $C M(\mathbb{Z})$ generated as a free Abelian group by the monomials $c \mu(T)$.

\section{Algebra $H^{*}(C M)$}

In this section, we use the combinatorial description of algebra $C M$ from the previous section to give a combinatorial description of algebra $H^{*}(C M)$.

In the rest of the paper, we will work much with homology of lattices. Let us introduce the notation that we will use. A lattice $L$ has two operations: the least upper bound $\vee$ (join) and the greatest lower bound $\wedge$ (meet). For any subset $\sigma \subseteq L$ put

$$
\bigvee(\sigma)=\bigvee_{A \in \sigma} A
$$

The smallest and largest elements of a lattice will be denoted by 0 and 1 respectively unless more specific symbols are appropriate. The set of all the atoms of $L$ will be denoted by $\mathfrak{A}=\mathfrak{A}(L)$. An arbitrary linear ordering is fixed on this set and all subsets of atoms will be ordered by the induced order. For every $T \in L$ the set of complements of $T$ is $\mathcal{C}(T)=\{S \in L \mid S \vee T=1, S \wedge T=0\}$.

With an arbitrary poset $P$ one can associate the abstract simplicial complex $F(P)$ on $P$ whose simplexes are all the flags of $F(P)$. For a lattice $L$, one also considers another abstract simplicial complex, the atomic complex $\Delta(L)$ on $\mathfrak{A}$. Its simplexes are the subsets $\sigma \subseteq \mathfrak{A}$ with $\bigvee(\sigma)<1$. Let $L_{0}=L \backslash\{0,1\}$. Then $\Delta(L)$ is homotopy equivalent to $F\left(L_{0}\right)$ (see [2] and Lemma 6.1] below) whence their 
homology are isomorphic. This homology is called the homology of $L$ and denoted by $H_{*}(L)$. For any simplicial complex $\Delta$ we denote by $C(\Delta)$ its chain complex over $\mathbb{Q}$ augmented by $\mathbb{Q}$ in degree -1 (although many results below hold over $\mathbb{Z}$ ).

We need to fix a particular homotopy equivalence of the chain complexes $C(\Delta(L))$ and $C\left(F\left(L_{0}\right)\right)$. For that denote by $\Delta^{\prime}(L)$ the barycentric subdivision of $\Delta(L)$ and identify $\Delta^{\prime}(L)$ with $F(Q)$ where $Q$ is the poset of all nonempty simplexes of $\Delta(L)$ ordered by inclusion. Denote by $\beta$ the standard homotopy equivalence $\beta: C(\Delta(L)) \rightarrow C(F(Q))$ defined by

$$
\beta\left(\left\{A_{1}, \ldots, A_{p}\right\}\right)=\sum_{\delta} \operatorname{sign} \delta\left(\left\{A_{\delta(1)}\right\},\left\{A_{\delta(1)}, A_{\delta(2)}\right\}, \ldots,\left\{A_{\delta(1)}, \ldots, A_{\delta(p)}\right\}\right)
$$

where $\left\{A_{1}, \ldots, A_{p}\right\}$ is an arbitrary simplex of $\Delta(L)$ (ordered according to the fixed order on $\mathfrak{A})$ and the summation is taken over all the permutations $\delta$ of rank $p$. Also define the order preserving map $\gamma: Q \rightarrow L_{0}$ via $\gamma(\sigma)=\bigvee(\sigma)$ for $\sigma \in \Delta(L)$ and keep the same symbol for the respective chain map $C(F(Q)) \rightarrow C\left(F\left(L_{0}\right)\right)$. The map $\gamma$ is a homotopy equivalence since for every $C \in L_{0}$ the poset $\gamma^{-1}\left(\left\{B \in L_{0} \mid B \leq C\right\}\right)$ has the unique maximal element $\{A \in \mathfrak{A}(L) \mid A \leq C\}$ whence is contractible (e.g., see [14]). Thus we can register the following lemma.

Lemma 6.1. For a lattice $L$ the group homomorphism $f_{L}=\gamma \beta: C(\Delta(L)) \rightarrow$ $C\left(F\left(L_{0}\right)\right)$ given on atomic simplexes by

$$
f_{L}\left(A_{1}, \ldots, A_{p}\right)=\sum_{\delta} \operatorname{sign} \delta\left(A_{\delta(1)} \leq A_{\delta(1)} \vee A_{\delta(2)} \leq \cdots \leq \bigvee_{i=1}^{p} A_{\delta(i)}\right),
$$

where the summation is taken over all the permutations $\delta$ of rank $p$ and every flag with repetitions considered to be 0 , is a homotopy equivalence of chain complexes.

Let us introduce several more pieces of notation. For every $A, B \in L$ such that $B \leq A$ there is the lattice (closed interval) $[B, A]=\{C \in L \mid B \leq C \leq A\}$ whose homology is denoted simply by $H_{*}(B, A)$. The set of atoms of $[0, A]$ we denote by $\mathfrak{A}(A)$, the flag complex of $[0, A]_{0}=(0, A)$ by $F(A)$ and the atomic complex of $[0, A]$ by $\Delta(A)$. For any simplicial complex $\Delta$ we denote by $\mathcal{S}(\Delta)$ its suspension. For any simplicial complexes $\Delta_{1}$ and $\Delta_{2}$ we denote by $\Delta_{1} * \Delta_{2}$ their join. Also we use $\bar{\Delta}$ for the simplex on all the vertices of $\Delta$. For every $A, B \in L$ we denote by $\Delta(A, B)$ the full subcomplex of $\Delta(A \vee B)$ on the vertex set $\mathfrak{A}(A, B)=\mathfrak{A}(A) \cup \mathfrak{A}(B)$. The joins of all subsets of $\mathfrak{A}(A, B)$ form a sublattice of $[0, A \vee B]$ that we denote by $L_{A, B}$ and we write $i(A, B)$ for the inclusion $L_{A, B} \subseteq[0, A \vee B]$. We denote by $F(A, B)$ the flag complex of $L_{A, B}$.

Now we return to the poset $X$ of subspaces. When we apply to $X$ notation and results from lattice theory we always mean that 0 is adjoined to it.

Lemma 6.2. If $A, B \in X$ are such that $A \cap B=0$, then

$$
\Delta(A, B)=(\overline{\Delta(A)} * \Delta(B)) \cup(\Delta(A) * \overline{A(B)}) .
$$

Proof. Condition $A \cap B=0$ implies the similar condition on any subspaces of $A$ and $B$. In particular, for $A_{1} \leq A$ and $B_{1} \leq B$ we have $A_{1} \vee B_{1}=A \vee B$ only if $A_{1}=A$ and $B_{1}=B$. The result follows immediately.

Lemma 6.2 implies in particular that under the condition of the lemma, $\Delta(A, B)$ is homotopy equivalent to $\mathcal{S}(\Delta(A) * \Delta(B))$. To fix a homotopy equivalence it is 
convenient to pass to $\Delta(A, B)^{\prime}$. Denote the additional vertices of the suspended complex $\mathcal{S}(\Delta(A) * \Delta(B))$ by $a$ and $b$ and consider the chain map

$$
\phi=\phi(A, B): C(\mathcal{S}(\Delta(A) * \Delta(B))) \rightarrow C\left(\Delta(A, B)^{\prime}\right)
$$

defined as follows. First of all, it acts as $\beta$ on simplexes in $\Delta(A) * \Delta(B)$. Recall that the images under $\beta$ are flags of simplexes in $\Delta(A, B)$. Then $\phi$ maps $a$ and $b$ to the barycenters of $\overline{\Delta(A)}$ and $\overline{\Delta(B)}$ respectively, that are $\mathfrak{A}(A)$ and $\mathfrak{A}(B)$. Notice that the latter sets of atoms can, as simplexes in $\Delta(A, B)$ (cf. Lemma 6.2), be included into the flags that occur as images under $\beta$.

For this map to be well-defined we need to specify that the vertices of the suspension are taken last among vertices of simplexes containing them. For instance, if $\left\{A_{1}\right\} \in \Delta(A),\left\{B_{1}\right\} \in \Delta(B)$ and $A_{1}<B_{1}$ in the ordering on atoms, then

$$
\begin{aligned}
\phi\left(\left\{A_{1}, B_{1}, a\right\}\right)= & \left(\left\{A_{1}\right\} \subset\left\{A_{1}, B_{1}\right\} \subset \mathfrak{A}(A) \cup\left\{B_{1}\right\}\right) \\
& -\left(\left\{A_{1}\right\} \subset \mathfrak{A}(A) \subset \mathfrak{A}(A) \cup\left\{B_{1}\right\}\right) \\
& -\left(\left\{B_{1}\right\} \subset\left\{A_{1}, B_{1}\right\} \subset \mathfrak{A}(A) \cup\left\{B_{1}\right\}\right) .
\end{aligned}
$$

Lemma 6.3. The map $\phi$ is a homotopy equivalence of chain complexes.

This follows from Lemma 6.2 and from the fact that $\phi$ is really an algebraic version of the natural homotopy equivalence of realizations of the involved abstract complexes.

Finally we need a result from elementary algebraic topology. It is well-known but hard to find in the literature. It follows immediately from an exact homology sequence for the join of two complexes (11], p. 373), the isomorphism for the homology of the suspension ([1], p. 47), and the Künneth formula. Specialized for our situation, the result is as follows.

Lemma 6.4. For every $A, B \in X$ the group homomorphism

$$
\psi=\psi(A, B): C(\Delta(A)) \otimes C(\Delta(B)) \rightarrow C(\mathcal{S}(\Delta(A) *(B))),
$$

given by

$$
\sigma \otimes \tau \mapsto(-1)^{\operatorname{dim} \tau} \operatorname{sign} \varepsilon(\sigma, \tau)(\sigma \cup \tau \cup\{b\}-\sigma \cup \tau \cup\{a\})
$$

for $\sigma \in \Delta(A)$ and $\tau \in \Delta(B)$, is a quasi-isomorphism of degree +2 of chain complexes. Here $\varepsilon(\sigma, \tau)$ is the shuffle of a $\sigma \cup \tau$ putting all the elements of $\tau$ after elements of $\sigma$.

It is important to keep in mind that all our chain complexes are augmented and defined over a field. The sign for $\psi(\sigma \otimes \tau)$ in Lemma 6.4 is chosen so that the diagram in the proof of Theorem 6.6 will be commutative.

If we put $\alpha(A, B)=\alpha=\phi(A, B) \psi(A, B)$ and keep in mind that the poset $X_{A, B}$ is homotopy equivalent to the face poset of $\Delta(A, B)$ then Lemmas 6.3 and 6.4 and the Künneth formula imply the following.

Corollary 6.5. The map

$$
\alpha_{*}: \widetilde{H}_{r}((0, A), \mathbb{Q}) \otimes \widetilde{H}_{s}((0, B), \mathbb{Q}) \rightarrow \widetilde{H}_{r+s+2}\left(X_{A, B}, \mathbb{Q}\right)
$$

is an isomorphism for every $r$ and $s$.

Now we are ready to describe $H^{*}(C(\mathcal{A}), \mathbb{Q})=H^{*}(C M)$. 
Theorem 6.6. (i) For every $p$ we have isomorphisms

$$
\widetilde{H}^{p}(C(\mathcal{A}), \mathbb{Q}) \approx \widetilde{H}^{p}(C M) \approx \bigoplus_{A \in X} \widetilde{H}_{2 \operatorname{dim} A-p-2}((0, A), \mathbb{Q}) .
$$

(ii) Under the isomorphism from (i) the multiplication on $H^{*}(C(\mathcal{A}), \mathbb{Q})$ is given by the compositions

$$
\widetilde{H}_{r}((0, A), \mathbb{Q}) \otimes \widetilde{H}_{s}((0, B), \mathbb{Q}) \stackrel{\alpha_{*}}{\longrightarrow} \widetilde{H}_{r+s+2}\left(X_{A, B}, \mathbb{Q}\right) \stackrel{i_{*}}{\longrightarrow} \widetilde{H}_{r+s+2}((0, A \vee B), \mathbb{Q})
$$

if $A \cap B=0$ (equivalently $\operatorname{dim}(A \vee B)=\operatorname{dim} A+\operatorname{dim} B$ ) and 0 otherwise.

Proof. (i) has been proved before in section 4 and is included here just for completeness. Thus we need to prove (ii) only.

In the rest of the proof all homology groups have the rational coefficients and we suppress $\mathbb{Q}$. We know from Corollary 5.2 that the multiplication on $H_{*}(0, A) \otimes$ $H_{*}(0, B)$ is 0 unless $A \cap B=0$. Hence let us fix $A, B \in X$ such that $A \cap B=0$. Then consider the following diagram

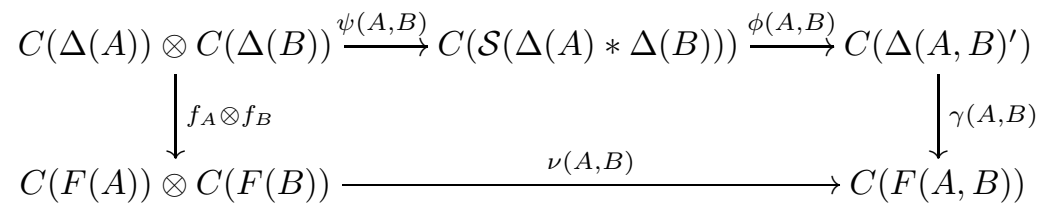

where $\nu(A, B)$ is the shuffle multiplication of flags from the previous section and $\gamma(A, B)$ is the natural map (introduced before Lemma 6.1) for the lattice $X_{A, B}$. Notice that before one applies the shuffle multiplication to two flags from $(0, A)$ and $(0, B)$ one should augment them by $A$ and $B$ respectively and then delete $A \vee B$ from all flags in the support of the product. A straightforward check shows that the diagram commutes. Since the vertical maps are homotopy equivalences, passing to the homology completes the proof.

The statement (ii) of Theorem 6.6 makes sense over $\mathbb{Z}$. I make the following conjecture.

Conjecture 6.7. Theorem 6.6 (ii) holds for the ring $H^{*}(C(\mathcal{A}), \mathbb{Z})$ (using integer homology of the posets). In particular, this ring is defined by the poset structure on $X$ (the "combinatorics" of the arrangement) and the dimensions of its elements.

\section{Geometric lattices}

In this section, we suppose that $X$ (augmented by 0 ) is a geometric lattice, i.e., every element is the join of a set of atoms, for every $A \in X$ all maximal flags ending on $A$ have the same length (that is the number of elements minus 1 ) denoted by $\operatorname{rk} A$ and $\operatorname{rk}(A \vee B)+\operatorname{rk}(A \wedge B) \leq \operatorname{rk} A+\operatorname{rk} B$ for every $A, B \in X$. In this case we are able to give a more explicit description of $H^{*}(C(\mathcal{A})$ ) (all cohomology and homology in this and the following section are over $\mathbb{Q})$.

By Folkman's theorem [7] $\widetilde{H}_{p}(0, A) \neq 0$ only for $p=\operatorname{rk} A-2$.

The following observation will be helpful.

Lemma 7.1. For every $A, B \in X$ such that $A \cap B=0$ we have $\operatorname{rk}(A \vee B)=$ rk $A+\operatorname{rk} B$. 
Proof. Put $k=\operatorname{rk} A, l=\operatorname{rk} B$ and fix two flags in $X$

$$
0=A_{0}<A_{1}<A_{2}<\cdots<A_{k}=A, 0=B_{0}<B_{1}<B_{2}<\cdots<B_{l}=B .
$$

For any $i, 0 \leq i \leq l$, we have $A \cap B_{i} \subseteq A \cap B=0$ whence $\operatorname{dim}\left(A+B_{i}\right)=$ $\operatorname{dim} A+\operatorname{dim} B_{i}$. Thus we have the flag in $X$

$$
0<A_{1}<A_{2}<\cdots<A_{k}=A<A+B_{1}<A+B_{2}<\cdots<A+B_{l}=A+B
$$

which implies $\operatorname{rk}(A \vee B) \geq \operatorname{rk} A+\operatorname{rk} B$. The opposite inequality follows immediately from the inequality for rank in geometric lattice.

Thus the multiplication $\nu(A, B)$, which is 0 if $A \cap B \neq 0$, is a map

$$
\nu(A, B): \widetilde{H}_{\mathrm{rk} A-2}(0, A) \otimes \widetilde{H}_{\mathrm{rk} B-2}(0, B) \rightarrow \widetilde{H}_{\mathrm{rk}(A \vee B)-2}(0, A \vee B)
$$

otherwise.

To describe multiplication (7.1) more precisely and give a presentation of $H^{*}(C(\mathcal{A}))$ we use the well known basis of the homology of geometric lattices (e.g., see [13], p. 144). Recall that a set $\sigma$ of atoms of a geometric lattice is called independent if $\operatorname{rk} \bigvee(\sigma)=|\sigma|$. Clearly every subset of an independent set is again independent. Suppose $A \in X$ and $\operatorname{rk} A=p$. Fix an arbitrary linear order on the set of atoms $\mathcal{A}(X)$. Then for any independent subset $\sigma=\left\{A_{1}, \ldots, A_{p}\right\} \subseteq \mathcal{A}(A)$ the cycle $\partial(\sigma)=\sum_{i=1}^{p}(-1)^{i} \sigma \backslash\left\{A_{i}\right\}$ of $\Delta(A)$ defines a nonzero element $\zeta_{\sigma} \in \widetilde{H}_{p-2}(0, A)$ and all these elements generate the space. If a set $\sigma$ is dependent we will use the symbol $\zeta_{\sigma}$ sometimes too but its value will be always 0 . In general the elements $\zeta_{\sigma}$ are not linearly independent over $\mathbb{Z}$ even for independent sets $\sigma$. Linear relations among them are generated by

$$
\sum_{j=1}^{p+1}(-1)^{j} \zeta_{\tau \backslash\left\{B_{j}\right\}}
$$

for every dependent set $\tau=\left\{B_{1}, \ldots, B_{p+1}\right\} \subseteq \mathcal{A}(A)$. Lemma 7.1 implies that if $A \cap B=0$ and $\sigma \subseteq \mathcal{A}(A)$ and $\tau \subseteq \mathcal{A}(B)$ are independent sets, then the set $\sigma \cup \tau \subseteq \mathcal{A}(A \vee B)$ is again independent.

Now Theorem 6.6 implies the following result.

Proposition 7.2. Suppose $X$ is a geometric lattice. Then the algebra $H^{*}(C(\mathcal{A}))$ is generated by $\zeta_{\sigma} \in H^{q}(C(\mathcal{A}))$ where $\sigma$ runs through all the independent sets of atoms of $X$ and $q=2 \operatorname{dim} \bigvee(\sigma)-|\sigma|$. Besides the linear relations (7.2), only other generating relations are

$$
\zeta_{\sigma} \zeta_{\tau}=\operatorname{sign} \varepsilon(\sigma, \tau) \zeta_{\sigma \cup \tau}
$$

if $\bigvee(\sigma) \cap \bigvee(\tau)=0$ (where $\varepsilon(\sigma, \tau)$ is the permutation of $\sigma \cup \tau$ putting all the elements of $\tau$ after elements of $\sigma)$ and

$$
\zeta_{\sigma} \zeta_{\tau}=0
$$

otherwise.

Remark 7.3. (i) In general, relations (7.2) are not linearly independent. One can easily construct a linear resolution of the space of generators $\zeta_{\sigma}$ and recover Folkman's formula for the dimension of $H_{*}(0, A)$ from [7].

(ii) Proposition 7.2 can be used to prove that the space $C(\mathcal{A})$ is formal in this case. 
(iii) In the case when $X$ is not only geometric but Boolean all the homologies in (7.1) are 1-dimensional and the multiplication, if nontrivial, is clearly an isomorphism (cf. 6]). In general the multiplication is not an isomorphism.

\section{Cohomology Rings of " $k$-EQUAL" manifolds}

In this section, for each pair $(n, k)$ of integers with $2 \leq k \leq n$ and for each $k$-subset $\omega \subseteq\{1,2, \ldots, n\}$ we consider the subspace $V(\omega) \subset \mathbf{C}^{n}=\left\{\left(x_{1}, \ldots, x_{n}\right)\right\}$ defined by the system of equations $\sum_{i \in \omega} x_{i}=0$ and $x_{j}=0$ for $j \notin \omega$. The set of all $V(\omega)$ (for fixed $n$ and $k$ ) is denoted by $\mathcal{A}_{n, k}^{\circ}$. Notice that the annihilator $V(\omega)^{0}$ of $V(\omega)$ is defined in $\left(\mathbf{C}^{n}\right)^{*}=\left\{\left(z_{1}, \ldots, z_{n}\right)\right\}$ by the equations $z_{1}=z_{j}$ for $i, j \in \omega$. That justifies the name $k$-equal manifold introduced in [3] for $M_{n, k}=C\left(\mathcal{A}_{n, k}^{\circ}\right)$.

The linear structure of $H^{*}\left(M_{n, k}\right)$ has been studied in detail in 3]. Completing $\mathcal{A}_{n, k}^{\circ}$ by the sums of its elements one obtains the lattice $\Pi_{n, k}$ that is (naturally isomorphic to) the lattice of all partitions of the set $\{1, \ldots, n\}$ whose blocks consist of either 1 element (trivial blocks) or at least $k$ elements (nontrivial blocks). In the rest of the paper, we will regard elements of $\Pi_{n, k}$ both as subspaces and partitions. Theorem 1.5 of [3] asserts that $\widetilde{H}_{p}\left(\Pi_{n, k}\right) \neq 0$ if and only if $p=n-3-t(k-2)$ for some integer $t$ such that $1 \leq t \leq\lfloor n / k\rfloor$.

Although the lattice $\Pi_{n, k}$ is not geometric in general, it has a similar property; there exist generators of its homology groups represented each by a simplicial sphere in the atomic complex. To prove that we need to use a recursive construction from 3. hence to include into consideration more general lattices introduced there. For any nonnegative integers $n, k$, and $l$ such that $n, k \geq 2$ and either $k \leq n$ or $l>0$, define $\Pi_{n, k}(l)$ as the family of all partitions of the set $\{1, \ldots, n\}$ such that every block $b$ satisfies at least one of the following requirements:

(i) $|b|=1$,

(ii) $|b| \geq k$,

(iii) $b \cap\{1, \ldots, l\} \neq \emptyset$.

Ordered by refinement $\Pi_{n, k}(l)$ becomes a lattice and $\Pi_{n, k}(0)=\Pi_{n, k}$.

First we need to recall the recursive construction used in [3] for the homology groups of $\Pi_{n, k}(l)$. Suppose $n \geq 3$ and $k, l \leq n-1$. Let $T$ be a coatom of $\Pi_{n, k}(l)$ having the nontrivial block $b=\{1, \ldots, n-1\}$. Clearly there are two types of complements $S$ of $T$, both are atoms whose unique nontrivial blocks we denote by $b_{1}$. Either $\left|b_{1}\right|=k, n \in b_{1}$, and $b_{1} \cap\{1, \ldots, l\}=\emptyset$ or $b_{1}=\{i, n\}$ where $i \leq l$. In the former case the poset $[S, 1]$ is isomorphic to $\Pi_{n-k+1, k}(l+1)$, in the latter case it is isomorphic to $\Pi_{n-1, k}(l)$. The subposet $\left(\Pi_{n, k}(l)\right)_{0}=(0,1)$ of $\Pi_{n, k}(l)$ is homotopy equivalent to the wedge of suspensions of the posets $(S, 1)$ where $S$ runs over $\mathcal{C}(T)$ (see details and references in [3]). In particular, taking a basis of homology for each $[S, 1]$ and applying suspension one obtains a basis of homology of $\Pi_{n, k}(l)$. Notice that for each choice of $S$ the homotopy type of the suspension of $(S, 1)$ can be realized as a subposet of $(0,1)$. For instance, one can take as such a realization $(S, 1) \cup\{S, T\}$ to which at least one atom $A_{0} \leq T \wedge A$ for each $A \in(S, 1)$ is added. We will call any of these (homotopy equivalent to each other) posets the suspension of $(S, 1)$ with vertices $S$ and $T$. We will also use the same term for the suspension of homology classes of $(S, 1)$.

The result of the above recursive construction for $\Pi=\Pi_{n, k}$ can be described as follows. Fix a maximal flag $0=S_{0}<S_{1}<S_{2}<\cdots<S_{p+1}<1$ in $\Pi$ and a sequence of elements $T_{1}, T_{2}, \ldots, T_{p}$ such that for every $i=1,2, \ldots, p$ the element 
$T_{i}$ has a nontrivial block of size $n-1, T_{i} \vee S_{i}=1$ and $T_{i} \wedge S_{i}=S_{i-1}$. A pair $\left(S^{\prime}, S_{p+1}\right)$, where $S^{\prime}$ is any atom of $\left[S_{p}, 1\right]$ different from $S_{p+1}$, defines an element $\alpha_{p} \in \widetilde{H}_{0}\left(S_{p}, 1\right)$. Applying recursively suspensions with vertices $S_{i}$ and $T_{i}$ one obtains a sequence of $\alpha_{i} \in \widetilde{H}_{p-i}\left(S_{i}, 1\right)$. Then elements $\alpha_{0}$ taken for all the above data generate $\widetilde{H}_{p}(\Pi)$. The condition $\alpha_{0} \neq 0$ puts restrictions on $S_{p}$ and $S_{p+1}$. In fact, the variety of possibilities reduces to the following four cases (cf. Lemma 4.4 from $[3])$.

(a) $n=k+1$ and $p=0$

(b) $S_{p}$ has only one nontrivial block of $n-k$ elements and $S_{p+1}$ has besides the complementary block (of $k$ elements);

(c) $S_{p}$ has three blocks all together exactly one of which is nontrivial $(k \geq 3)$;

(d) $S_{p}$ has three blocks all together at least two of which are nontrivial.

Now we are ready to study the multiplication on local homology of П. For every $p$ consider the homomorphism

$$
\xi_{p}: \bigoplus_{\{A, B\}} \widetilde{H}_{p}\left(\Pi_{A, B}\right) \rightarrow \widetilde{H}_{p}(\Pi)
$$

induced by embeddings where $\{A, B\}$ runs through all the subsets of $\Pi$ such that $A$ and $B$ are one-block partitions, $A \wedge B=1$ and $A \cap B=0$. The last two conditions can be substituted by $b_{A} \cup b_{B}=\{1, \ldots, n\}$ and $\left|b_{A} \cap b_{B}\right|=0$ where $b_{A}$ and $b_{B}$ are the nontrivial blocks of $A$ and $B$ respectively. Recall that the maximal $p$ with $\widetilde{H}_{p}(\Pi) \neq 0$ is $p=n-k-1$. It is easy to see that $\xi_{n-k-1}=0$ since its domain is 0 . On the other hand we have the following.

Lemma 8.1. For $p<n-k-1$ the homomorphism $\xi$ is surjective.

Proof. It suffices to fix generators of $\widetilde{H}_{p}(\Pi)(p<n-k-1)$ and for each $\alpha$ of them find $A$ and $B$ with required properties and such that $\alpha \in \operatorname{Im} \xi$.

To fix an element $\alpha$ of a generating set we fix sequences $\left(S_{i}\right)$ and $\left(T_{i}\right)$ as above and, using suspension, construct recursively $\alpha_{i} \in \widetilde{H}_{p-i}\left(S_{i}, 1\right)$ starting from $\alpha_{p}$ and finishing at $\alpha_{0}=\alpha$. The condition $p<n-k-1$ restricts possibilities for $S_{p}$ to (b) and (d) only. In case (b), we take as $S^{\prime}$ any atom of $\left[S_{p}, 1\right]$ different from $S_{p+1}\left(\alpha_{p}\right.$ is essentially unique in this case). In case (d), we are a little more careful. Each $S_{i}$ has a block that contains the nontrivial block of $S_{1}$. We call this block of $S_{i}$ distinguished. While choosing an atom $S^{\prime}$ we avoid repetition of the distinguished block of $S_{p}$, i.e. if this block is a proper subset of another block in $S_{p+1}$, then in $S^{\prime}$ this block is not a proper subset of any block. Clearly such elements $\alpha_{p}$ generate $\widetilde{H}_{0}\left(S_{p}, 1\right)$.

Now we denote by $A_{p}$ the element of the pair $\left\{S_{p+1}, S^{\prime}\right\}$ that contains the distinguished block of $S_{p}$ as a proper subset of another block and by $B_{p}$ the other element of the pair. Our goal is to construct recursively a sequence $\left(A_{i}, B_{i}\right)$ $(i=p, p-1, \ldots, 0)$ with the following properties.

(i) $A_{i}, B_{i}>S_{i}$ and each has only one nontrivial block ( $b_{i}^{\prime}$ and $b_{i}^{\prime \prime}$ respectively) with respect to $S_{i}$;

(ii) $b_{i}^{\prime} \cap b_{i}^{\prime \prime}$ is a block of $S_{i}$;

(iii) $A_{i} \vee B_{i}=1$;

(iv) For $i>0$ only $A_{i}$ (and not $B_{i}$ ) has a block that contains the distinguished block of $S_{i}$ as its proper subset;

(v) $\alpha_{i}$ is in the image of $\widetilde{H}_{i}\left(\left[S_{i}, 1\right]_{A_{i}, B_{i}}\right)$ in $\widetilde{H}_{i}\left(S_{i}, 1\right)$. 
Notice that $A_{p}$ and $B_{p}$ satisfy the conditions (i)-(v). Now suppose that for some $i, 0<i \leq p, A_{i}$ and $B_{i}$ satisfying (i)-(v) are constructed. The construction of $A_{i-1}$ and $B_{i-1}$ depends on $b_{i}^{\prime} \cap b_{i}^{\prime \prime}$. Denote by $b_{i}$ the unique block of $S_{i}$ that is nontrivial with respect to $S_{i-1}$ and let $b_{i}=\bigcup_{j} b_{i j}$ be its partition into blocks of $S_{i-1}$. If $b_{i}^{\prime} \cap b_{i}^{\prime \prime} \neq b_{i}$, then define $A_{i-1}$ and $B_{i-1}$ as having the nontrivial blocks $b_{i-1}^{\prime}=b_{i}^{\prime}$ and $b_{i-1}^{\prime \prime}=b_{i}^{\prime \prime}$ respectively, relative to $S_{i-1}$. If $b_{i}^{\prime} \cap b_{i}^{\prime \prime}=b_{i}$, then define $b_{i-1}^{\prime \prime}$ as in the previous case and put $b_{i-1}^{\prime}=\left(b_{i}^{\prime} \backslash b_{i}\right) \cup b_{i j}$ for some $j$ such that $b_{i j}$ is a subset of the nontrivial block of $T_{i}$. The property (iv) of $A_{i}$ and $B_{i}$ guarantees that $A_{i-1} \in \Pi$. In any case the conditions (i)-(iv) for $i-1$ are satisfied by construction and we only need to check $(\mathrm{v})$.

To simplify notation put $P_{i}=\left[S_{i}, 1\right]_{A_{i}, B_{i}}$ for every $i$ and denote the element not contained in the nontrivial block of $T_{i}$ by $a_{i}$. First notice that by construction either $A_{i-1}$ or $B_{i-1} \leq T_{i}$. Suppose that $A_{i-1} \leq T_{i}$ since the other case can be handled similarly (even more easily). This implies that $b_{i} \subset b_{i-1}^{\prime \prime}$. Then let us prove that $T_{i} \in P_{i-1}$. Since $\Pi$ is atomic we have $A_{i-1}, B_{i-1} \in P_{i-1}$. Define $B^{\prime}$ as the one-block partition with the nontrivial block $b_{i-1}^{\prime \prime} \backslash\left\{a_{i}\right\}$. Since $b_{i} \subset b_{i-1}^{\prime \prime}$ we have $B^{\prime} \in \Pi$ whence $B^{\prime} \in P_{i-1}$. Since $A_{i-1} \vee B^{\prime}=T_{i}$ we have $T_{i} \in P_{i-1}$.

Our second observation is that $P_{i} \subset P_{i-1}$. To see that it suffices to prove that any atom $C$ of $\left[S_{i}, 1\right]$ such that $C \leq A_{i}$ or $C \leq B_{i}$ is in $P_{i-1}$. Denote the only nontrivial with respect to $S_{i}$ block of $C$ by $b_{C}$. If $b_{C} \not \supset b_{i}$, then the atom $C_{0}$ of $\left[S_{i-1}, 1\right]$ having only nontrivial block $b_{C}$ with respect to $S_{i-1}$ is in $P_{i-1}$ and $C_{0} \vee S_{i}=C$. Suppose $b_{C} \supset b_{i}$ and $C \leq A_{i}$. Then $C_{0}=C \wedge A_{i-1}$ is an atom of $P_{i-1}$ and again $C_{0} \vee S_{i}=C$. The case $C \leq B_{i}$ is similar. In any case we have $C \in P_{i-1}$.

Summing up our above observations we see that $P_{i-1}$ contains the suspension of $P_{i}$ with vertices $S_{i}$ and $T_{i}$ (cf. [2] or Proposition 2.3 and p. 290 of [3]). Since by the inductive hypothesis (v) holds for $i$ we obtain it for $i-1$.

Putting $A=A_{0}$ and $B=B_{0}$ we complete the proof.

In order to exhibit more explicit generators of $H^{*}\left(M_{n, k}\right)$ we use the atomic complexes as in the previous section. We will always assume that the atoms are linearly ordered and all sets of atoms are provided with the induced ordering. By $C(L)$ we denote the chain complex of the abstract simplicial complex formed by all sets of atoms in $L$; the differential in $C(L)$ is denoted by $\partial$.

Definition 8.2. A set $\sigma$ of atoms of a lattice $L$ is called independent if $\bigvee(\tau) \neq$ $\bigvee(\sigma)$ for every proper subset $\tau$ of $\sigma$. It is called essential if it is not a simplex in the atomic complex $\Delta(L)$, i.e. if $\bigvee(\sigma)=1$. An element $\omega$ of $C(L)$ is called a relater in $L$ if $\partial \omega$ can be represented as a linear combination of independent essential sets and simplexes of the atomic complex, i.e., sets bounded by elements less than 1 .

Any set $\sigma=\left\{A_{1}, A_{2}, \ldots, A_{p}\right\}$ of atoms of $L$, that is either a simplex in $\Delta(L)$ or independent, defines the cycle

$$
\partial \sigma=\sum_{i=1}^{p}(-1)^{i} \sigma \backslash\left\{A_{i}\right\} \in C_{p-2}(\Delta(L)) .
$$

Denote its homology class by $\zeta_{\sigma}$. If $\sigma$ is a simplex of $\Delta(L)$, then $\zeta_{\sigma}=0$. Any relater $\omega$ with $\partial \omega=\sum_{i} a_{i} \omega_{i}+\sum_{j} b_{j} \omega_{j}^{\prime}$ where $a_{i}, b_{j} \in \mathbb{Z}, \omega_{i}$ are independent essential sets and $\omega_{j}^{\prime}$ are simplexes in $\Delta(L)$ defines the linear relation $\sum_{i} a_{i} \zeta_{\omega_{i}}=0$. This motivates the following. Denote by $G_{p}$ the linear space generated by symbols $\eta_{\sigma}$ where $\sigma$ is either a simplex of $\Delta(L)$ or an independent essential set of atoms of 
$L$ and $|\sigma|=p+2$. Denote by $R_{p}$ the subspace of $G_{p}$ generated by the elements $\eta_{\sigma}$ where $\sigma$ is a simplex in $\Delta(L)$, and let $r_{\omega}=\sum_{i} a_{i} \eta_{\omega_{i}}$ where $\omega$ is a relator as above and $|\omega|=p+3$.

Fix $n, k, l$ such that $2 \leq k \leq n$ and $l \geq 0$ and put $\Pi=\Pi_{n, k}(l)$.

Lemma 8.3. (i) For every atom $S$ of $\Pi$ and a coatom $T$ with only one nontrivial block such that $T \in \mathcal{C}(S)$ there exists an injective map $\theta: \mathcal{A}([S, 1]) \rightarrow \mathcal{A}(\Pi) \backslash\{S\}$ such that $\bigvee(\operatorname{Im} \theta) \leq T$ and $\theta(\tau) \cup\{S\}$ is independent (essential) in $\Pi$ if and only if $\tau$ is independent (resp. essential) in $[S, 1]$.

Proof. Let $S$ and $T$ be as in the statement of the lemma. Consider the sets $W_{A}=$ $\mathcal{A}(T \wedge A)$ for $A \in \mathcal{A}([S, 1])$. It is easy to see that for every $B \leq T \wedge A$ we have

$$
B \vee S=A \text {. }
$$

This implies that the sets $W_{A}$ are pairwise disjoint. Choosing arbitrarily $\theta(A) \in W_{A}$ we obtain an injective map $\theta: \mathcal{A}([S, 1]) \rightarrow \mathcal{A}(\Pi) \backslash\{S\}$.

First let us prove the following property of $\theta$. For every $\tau \subseteq \mathcal{A}([S, 1])$

$$
\bigvee(\theta(\tau) \cup\{S\})=\bigvee(\tau)
$$

Indeed put $\tau^{\prime}=\theta(\tau \cup\{S\})$. If $D \geq B$ for every $B \in \tau$, then $D>S$ and $D>(T \wedge B)$ whence $D>A$ for every $A \in \tau^{\prime}$. Conversely if $D \geq A$ for every $A \in \tau^{\prime}$, then $D \geq(\theta(B) \vee S)=B$ for every $B \in \tau$.

Now it is easy to see that all the required properties of $\theta$ follow from construction and 8.1 .

Remark 8.4. The construction of a map $\theta$ in Lemma 8.3 uses an atom $S$ and a coatom $T$. We will say that $\theta$ is constructed with $S$ and $T$. Also it is clear from the construction that for every $\sigma \subseteq \mathcal{A}([S, 1])$ the class $\zeta_{\theta(\sigma)}$ is the suspension of $\zeta_{\sigma}$ with vertices $S$ and $T$.

To make the notation easier put $\Pi(l)=\Pi_{n, k}(l)$ for the length of the next proposition and denote the set of all essential independent subsets of atoms of $\Pi(l)$ by $\mathcal{I}=\mathcal{I}(\Pi(l))$.

Proposition 8.5. For every $p$, the linear map $\iota: G_{p} / R_{p} \rightarrow \widetilde{H}_{p}(\Pi(l))$ defined by $\iota\left(\eta_{\sigma}\right)=\zeta_{\sigma}$ for all $\sigma \in \mathcal{I}(\Pi(l)),|\sigma|=p+2$, is an isomorphism.

Proof. First we prove that $\iota$ is surjective. We apply induction on $p$ (for all $n, k$ and $l$ at the same time). As the base consider the case of $p=-1$. An essential 1-set $\sigma$ exists if and only if $(0,1)=\emptyset$ (i.e., $n=k, l=0$ or $n=2, l \geq 0)$ and then it is unique and independent. This is precisely the case when $\widetilde{H}_{-1}(\Pi(l)) \neq 0$ and $\zeta_{0}$ is a generator of this space.

Now suppose $p \geq 0$. If $k=n$, then the result is trivial. If $l \geq n$ or $n=2$, then $\Pi(l)$ is a geometric lattice and the result follows from Proposition 7.2. Thus we can assume that $n \geq 3, k, l \leq n-1$ and apply the above recursive construction for $H_{p}(\Pi(l))$. Fix a coatom $T$ with only nontrivial block of $n-1$ elements and atom $S$ such that $S \in \mathcal{C}(T)$. Recall that $(S, 1)$ is isomorphic to a poset $\Pi(l)_{n^{\prime}, k^{\prime}}\left(l^{\prime}\right)$ for some value of the parameters $n^{\prime}, k^{\prime}$ and $l^{\prime}$. Thus by the inductive hypothesis, one can find a basis of $\widetilde{H}_{p-1}(S, 1)$ consisting of classes $\zeta_{\sigma}$ where $\sigma$ runs through independent essential $p+1$-sets of atoms of $(S, 1)$. Using Lemma 8.3 (i) fix a map $\theta$ constructed with $S$ and $T$ and having property from that lemma. In particular, if $\sigma^{\prime}=\theta(\sigma)$, then $\sigma^{\prime}$ is independent and essential. By Remark $8.4 \zeta_{\sigma^{\prime}}$ is the suspension of $\zeta_{\sigma}$ with 
the vertices $S$ and $T$. Since the suspensions of generators of all spaces $H_{p-1}(S, 1)$ when $S$ runs through $\mathcal{C}(T)$ form a generating set of $H_{p}(\Pi(l))$ the proof is complete.

Now we prove that $\iota$ is an isomorphism. For that consider the factor complex $D=C(\Pi(l)) / C(\Delta(\Pi(l)))$. Notice that $D_{p}$ is generated by the essential sets of atoms of cardinality $p+1$ and the differential on $D$ is defined by

$$
d(\sigma)=\sum(-1)^{i} \sigma \backslash\left\{A_{i}\right\}
$$

where $\sigma=\left(A_{1}, \ldots, A_{p+1}\right)$ and the summation is taken over all $i$ such that

$$
\bigvee\left(\sigma \backslash\left\{A_{i}\right\}\right)=1
$$

In particular, an essential set is a cycle in $D$ if and only if it is independent. Applying the exact homological sequence of pair we see that the homomorphism $\delta$ : $\widetilde{H}_{p+1}(D) \rightarrow \widetilde{H}_{p}(C(\Delta))$ given by $\delta(z)=\partial z$ for any cycle $z \in D_{p}$ is an isomorphism. On the class $[\sigma]$ of an independent essential set $\sigma$ we have $\delta([\sigma])=\zeta_{\sigma}$. Thus the first part of the proof can be restated as follow: the classes of all the independent essential sets generate $H_{*}(D)$. On the other hand, $R_{p}$ is naturally isomorphic to the intersection $d\left(D_{p+2}\right) \cap\langle\mathcal{I}\rangle$ in $D_{p+1}$ and the result follows immediately.

The next lemma follows from Theorem [6.6 (ii) and [3], p. 296.

Lemma 8.6. Suppose $U \in \Pi_{n, k}$ and has precisely $s$ nontrivial blocks $b_{1}, \ldots, b_{s}$. Denote by $U_{i}$ the element of $\Pi_{n, k}$ having only one nontrivial block $b_{i}$. Then for every $p$ the repeated multiplication induces the isomorphism

$$
\bigoplus_{p_{1}+\cdots+p_{s}=p-2(s-1)} \widetilde{H}_{p_{1}}\left(0, U_{1}\right) \otimes \cdots \otimes \widetilde{H}_{p_{s}}\left(0, U_{s}\right) \cong \widetilde{H}_{p}(0, U) .
$$

Definition 8.7. Let $\sigma$ be a set of one-block partitions (e.g. atoms) from $\Pi_{n, k}$. Consider the collection $\mathcal{D}$ of all the subsets $\tau$ of $\sigma$ such that the nontrivial blocks of elements of $\tau$ are pairwise disjoint. Then the rank of $\sigma(\operatorname{rk}(\sigma))$ is the maximal cardinality of elements of $\mathcal{D}$.

Here is the main result of the section.

Theorem 8.8. (i) The space $H^{*}\left(M_{n, k}\right)$ is generated by the classes

$$
\zeta_{\sigma} \in H^{2 \operatorname{dim} U-|\sigma|}\left(M_{n, k}\right)
$$

where $\sigma$ runs through all the independent sets of atoms of $\Pi_{n, k}$ and $U=\bigvee(\sigma)$. The linear relations among $\zeta_{\sigma}$ are generated by $r_{\omega}$ for the relators $\omega$ of all $[0, A]$ $(A \in X)$.

(ii) The product $\zeta_{\sigma} \zeta_{\tau}=0$ if $\bigvee(\sigma) \cap \bigvee(\tau)=0$ (equivalently $\operatorname{dim} \bigvee(\sigma \cup \tau) \neq$ $\operatorname{dim} \bigvee(\sigma)+\operatorname{dim} \bigvee(\tau))$ and $\zeta_{\sigma} \zeta_{\tau}=\operatorname{sign} \varepsilon(\sigma, \tau) \zeta_{\sigma \cup \tau}$ otherwise where $\varepsilon(\sigma, \tau)$ is as above the shuffle of $\sigma \cup \tau$ putting all the elements of $\tau$ after elements of $\sigma$.

(iii) The linear relations from (i) imply that $\zeta_{\sigma}=0$ unless

$$
|\sigma|=n(\sigma)-\operatorname{rk}(\sigma)(k-2)-s,
$$

where $s$ is the number of nontrivial blocks in the partition $\bigvee(\sigma)$ and $n(\sigma)$ is the number of points in all these blocks. In particular, $\zeta_{\sigma} \zeta_{\tau}=0$ unless $\operatorname{rk}(\sigma \cup \tau)=$ $\operatorname{rk}(\sigma)+\operatorname{rk}(\tau)$.

(iv) As an algebra, $H^{*}\left(M_{n, k}\right)$ is generated by the classes $\zeta_{\sigma}$ for independent sets of atoms of rank 1 . 
Proof. (i) and (ii) follow immediately from Proposition 8.5 and Theorem 6.6

(iii) Let $\sigma$ be an independent set of atoms, $r=\operatorname{rk}(\sigma), U=\bigvee(\sigma)$ and $U_{1}, \ldots, U_{s}$ as in Lemma 8.6. Then $\sigma$ is a disjoint union of $\sigma_{i}(i=1, \ldots, s)$ where $\sigma_{i}$ is independent and $\bigvee\left(\sigma_{i}\right)=U_{i}$. Since $r=\sum_{i=1}^{s} \operatorname{rk}\left(\sigma_{i}\right)$ and $\zeta_{\sigma}=\zeta_{\sigma_{1}} \otimes \cdots \otimes \zeta_{\sigma_{s}}$ under identification (8.2) it suffices to consider the case where $s=1$, i.e. $U$ has only one nontrivial block of size $n(\sigma)$. It is easy to compute that

$$
n(\sigma) \geq|\sigma|+r(k-2)+1 .
$$

Now the recursive construction before Lemma 8.1 shows that we can assume without loss of generality that there exists a flag in the support of $f_{[0, U]}(\sigma)$ that is maximal in $(0, U]$. If this maximal flag has an element with $t$ nontrivial blocks and no elements with $t+1$ ones, then its length is

$$
t+(t-1)+(n(\sigma)-t k)=n(\sigma)-t(k-2)-1
$$

( $t$ steps to "create" new blocks, $t-1$ steps to glue blocks to each other and $n(\sigma)-t k$ steps to add a point to a block). Since for flags in the support of $f_{[0, U]}(\sigma)$ the maximal possible $t$ is $r$ we have

$$
|\sigma| \geq n(\sigma)-r(k-2)-1
$$

Comparing (8.4) and (8.5) we obtain 8.3).

The second part of (iii) follows since the equality $\operatorname{dim} \bigvee(\sigma)+\operatorname{dim} \bigvee(\tau)=$ $\operatorname{dim} \bigvee(\sigma \cup \tau)$ implies $n(\sigma \cup \tau)=n(\sigma)+n(\tau)-1$ for $\sigma$ and $\tau$ such that $\bigvee(\sigma)$ and $\bigvee(\tau)$ have only one nontrivial block each.

(iv) Fix an independent set $\sigma$ of atoms of rank $r>1$ and let again $s$ be the number of nontrivial blocks of $U=\bigvee(\sigma)$. Using Lemma 8.6 again we can assume that $s=1$. Suppose $\zeta_{\sigma} \neq 0$. Since $r>1$ we have from (iii) that $\zeta_{\sigma} \in \widetilde{H}_{p}(0, U)$ with

$$
p=|\sigma|-2=n(\sigma)-r(k-2)-3<n(\sigma)-k-1 .
$$

Now due to Lemma 8.1 and Theorem 6.6 (ii), $\zeta_{\sigma}$ is a linear combination of classes $\zeta_{\tau} \zeta_{\omega}= \pm \zeta_{\tau \cup \omega}$ where for each pair $(\tau, \omega)$ there exist $A$ and $B \in[0, U]$ such that $A \vee B=U, A \cap B=0, \zeta_{\tau} \in H_{q}(0, A)$ and $\zeta_{\omega} \in H_{p-q}(0, B)$ for some $q$. Since $\bigvee(\tau \cup \omega)=U$ we obtain from (iii) that $\operatorname{rk}(\tau \cup \omega)=r$. Using (iii) again we have $\operatorname{rk}(\tau)<r$ and $\operatorname{rk}(\omega)<r$ Now downward induction on $r$ completes the proof.

Remark 8.9. For any specified values of $n$ and $k$, the linear relations among $\zeta_{\sigma}$ can be used to find a basis of $\widetilde{H}_{p}(0, \bigvee(\sigma))(p=|\sigma|-2)$. In the case $\operatorname{rk}(\sigma)=1$ a basis is easy to describe in general which gives a generating set for the algebra $H^{*}\left(M_{n, k}\right)$.

Indeed let the only nontrivial block of $U=\bigvee(\sigma)$ be $b=\{1,2 \ldots, n(\sigma)\}$. Fix a subset $a$ of $b_{0}=b \backslash\{1\}$ with $|a|=k-1$ and put $\sigma(a)=\left\{A_{i} \mid i \in b \backslash a\right\}$ where $A_{i}$ is the atom whose only nontrivial block is $a \cup\{i\}$. Notice that $|\sigma|=n(\sigma)-k+1$ as it should be. Then the set (of cardinality $\left(\begin{array}{c}n(\sigma)-1 \\ k-1\end{array}\right)$ ) of all $\zeta_{\sigma(a)}$ is a basis of the subspace of $\widetilde{H}_{p}(0, U)$ generated by $\zeta_{\sigma}$ with $\operatorname{rk}(\sigma)=1$. 
Example. Let us consider the case where $n=6$ and $k=3$. The results for the additive structure of $H^{*}\left(M_{6,3}\right)$ are as the following table shows (cf. [3], p. 311).

$\begin{array}{cccccccc}\operatorname{dim} U & |\sigma| & p & \operatorname{rank}(\sigma) & s(\sigma) & n(\sigma) & q & \operatorname{dim} \widetilde{H}^{q}(M) \\ 2 & 1 & -1 & 1 & 1 & 3 & 3 & 20 \\ 3 & 2 & 0 & 1 & 1 & 4 & 4 & 45 \\ 4 & 3 & 1 & 1 & 1 & 5 & 5 & 36 \\ 5 & 4 & 2 & 1 & 1 & 6 & 6 & 10 \\ 4 & 2 & 0 & 2 & 2 & 6 & 6 & 10 \\ 5 & 3 & 1 & 2 & 1 & 6 & 7 & 10\end{array}$

In this table, $U=\bigvee(\sigma), p=|\sigma|-2$ is the dimension of the homology of $[0, U]$ generated by $\zeta_{\sigma}$ and $q$ is the respective dimension of the cohomology $H^{*}(M)$ with $M=M_{6,3}$. Notice that $H^{6}(M)$ is represented as $H^{6}(M)=H_{1}^{6}(M) \oplus H_{2}^{6}(M)$ where $H_{1}^{6}(M)$ is generated by $\zeta_{\sigma}$ with $|\sigma|=4$ and $\operatorname{rk}(\sigma)=1$ and $H_{2}^{6}(M)$ is generated by $\zeta_{\sigma}$ with $|\sigma|=2$ and $\operatorname{rk}(\sigma)=2$.

As a ring, $H^{*}(M)$ is generated by $H^{i}(M), i=3,4,5$, and $H_{1}^{6}(M)$. Using Remark 8.9 it is easy to exhibit a concrete set of algebra generators $\zeta_{\sigma}$. The multiplication $\nu$ is not zero only on $H^{3,3}=H^{3}(M) \otimes H^{3}(M)$ and $H^{3,4}=H^{3}(M) \otimes H^{4}(M)$. Due to Theorem 8.8, $\nu\left(\zeta_{\sigma} \otimes \zeta_{\tau}\right) \neq 0$ on $H^{3,3}$ only if $b_{A} \cap b_{B}=\emptyset$ where $\sigma=\{A\}, \tau=\{B\}$ and $b_{A}, b_{A}$ are respective nontrivial blocks of the atoms $A$ and $B$. This and the skew commutativity of $\nu$ give 10 linearly independent products $\zeta_{\sigma \cup \tau}$ in $H_{1}^{6}(M)$ that generate this whole space.

On $H^{3,4}$, consider the product $\pi=\zeta_{\sigma \cup \tau}$ where $\sigma=\{A\}$ and $\tau=\left\{B_{1}, B_{2}\right\}$ with $\left|b_{B_{1}} \cap b_{B_{2}}\right|=2$. Then Theorem 8.8 implies that $\pi \neq 0$ only if either $b_{B_{1}}$ or $b_{B_{2}}$ is disjoint with $b_{A}$ and in this case $\pi$ does not depend on the other atom in $\tau$. Again it is clear that there are 10 linearly independent products and they generate the whole space $H^{7}(M)$.

It may be instructive to exhibit an independent set of atoms $\sigma$ such that $\zeta_{\sigma}=0$. The simplest examples are given by $\sigma=\left\{B_{1}, B_{2}\right\}$ with $\left|b_{B_{1}} \cap b_{B_{2}}\right|=1$. For instance, if $\sigma=\{\{1,2,3\},\{1,4,5\}\}$ where the atoms are given by their nontrivial blocks, then $\omega=\sigma \cup\{\{1,2,4\}\}$ is a relater of $[0, \bigvee(\sigma)]$ and the relation $\partial \omega$ gives $\zeta_{\sigma}=0$. For a less obvious example one can take $\sigma=\{\{1,2,3\},\{3,4,5\},\{3,5,6\}\}$. Then $\operatorname{rk}(\sigma)=1$ and $3=|\sigma| \neq n(\sigma)-\operatorname{rk}(\sigma)(k-2)-s(\sigma)=4$. On the other hand, if $\omega=\sigma \cup\{\{1,3,5\}\}$, then it is a relator of $\Pi_{6,3}$ and the relation $r_{\omega}$ gives $\zeta_{\sigma}=0$.

\section{REFERENCES}

1. V. I. Arnold, The cohomology ring of the colored braid group, Mat. Zametki 5 (1969), 227-231 (Math. Notes 5 (1969), 138-140). MR 39:3529

2. A. Björner and J. Walker, A homotopy complementation formula for partially ordered sets, European J. Combin. 4 (1983), 11-19. MR 84f:06003

3. A. Björner and V. Welker, The homology of " $k$-equal" manifolds and related partition lattices, Adv. in Math. 110 (1995), 277-313. MR 95m:52029

4. E. Brieskorn, Sur les groupes de tresses, in Séminre Bourbaki 1971/72, Lecture Notes in Math., 317, Springer-Verlag, 1973, pp. 21-44. MR 54:10660

5. C. De Concini and C. Procesi, Wonderful models of subspace arrangements, Selecta Math. (N.S.) 1 (1995), 459-494. MR 97k:14013

6. E. M. Feichtner, Cohomology algebras of subspace arrangements and of classical configuration spaces, Cuvillier-Verlag, Göttingen, 1997 (Doctors Dissertation at TU, Berlin).

7. J. Folkman, The homology groups of a lattice, J. Math. Mech. 15 (1966), 631-636. MR 32:5557 
8. G. Gaiffi, Blow-ups and cohomology bases for De Concini-Procesi models of subspace arrangements, Selecta Math. (N.S.) 3 (1997), 315-333. MR 99d:52009]

9. M. Goresky and R. MacPherson, Stratified Morse Theory, Part III, Springer-Verlag, 1988. MR 90d:57039

10. J. Morgan, The algebraic topology of smooth algebraic varieties, Publ. Math. IHES 48 (1978), 137-204. MR 878m:55014

11. J. Munkres, Elements of algebraic topology, Addison-Wesley, Menlo Park, CA, 1984. MR 85m:55001

12. P. Orlik and L. Solomon, Combinatorics and topology of complements of hyperplanes, Invent. Math. 56 (1980), 167-189. MR 81e:32015

13. P. Orlik and H. Terao, Arrangements of hyperplanes, Springer-Verlag, Berlin, 1992. MR 94e:52014

14. D. Quillen, Homotopy properties of the poset of nontrivial p-subgroups of a group, Adv. in Math. 28 (1978), 101-128. MR 80k:20049

15. S. Yuzvinsky, Cohomology bases for the DeConcini-Procesi models of hyperplane arrangements and sums over trees, Invent. Math. 127 (1997), 319-335. MR 98m:14020

16. G. Ziegler and R. Živaljević, Homotopy types of subspace arrangements via diagrams of spaces, Math. Ann. 295 (1993), 527-548. MR 94c:55018

17. M. De Longueville and C. Schultz, The cohomology rings of complements of subspace arrangements, Math. Ann. 319 (2001), 625-646.

18. P. Deligne, M. Goresky, and R. MacPherson, L'algèbre de cohomologie du complément, dans un espace affine, d'une famille finie de sous-espaces affines, Michigan Math. J. 48 (2000), 121-136. CMP 2001:03

19. S. Yuzvinsky, Rational model of subspace complement on atomic complex, Publ. L'Institut Math. 66 (80) (1999), 157-164. CMP 2000:16

Department of Mathematics, University of Oregon, Eugene, Oregon 97403

E-mail address: yuz@math.uoregon.edu 